\title{
Termination Prediction for General Logic Programs
}

\author{
Yi-Dong Shen \\ State Key Laboratory of Computer Science, Institute of Software \\ Chinese Academy of Sciences, Beijing 100190, China \\ E-mail: ydshen@ios.ac.cn \\ Danny De Schreye and Dean Voets \\ Department of Computer Science, Celestijnenlaan 200 A, B-3001 Heverlee, Belgium \\ Email: \{danny.deschreye, dean.voets\}@cs.kuleuven.ac.be
}

\begin{abstract}
We present an approximation framework for attacking the undecidable termination problem of logic programs, as an alternative to current termination/non-termination proof approaches. We introduce an idea of termination prediction, which predicts termination of a logic program in case that neither a termination nor a non-termination proof is applicable. We establish a necessary and sufficient characterization of infinite (generalized) SLDNF-derivations with arbitrary (concrete or moded) queries, and develop an algorithm that predicts termination of general logic programs with arbitrary non-floundering queries. We have implemented a termination prediction tool and obtained quite satisfactory experimental results. Except for five programs which break the experiment time limit, our prediction is $100 \%$ correct for all benchmark programs of the Termination Competition 2007, of which eighteen programs cannot be proved by the existing state-of-the-art analyzers like AProVE07, NTI, Polytool and TALP.
\end{abstract}

Keywords: Logic programming, termination analysis, termination prediction.

\section{Introduction}

Termination is a fundamental problem in logic programming with SLDNF-resolution as the query evaluation mechanism [9, 18], which has been extensively studied in the literature (see, e.g., [10] for a survey and some recent papers $[2,6,11,13,16,23,27,31])$. Since the termination problem is undecidable, existing algorithms/tools either focus on computing sufficient termination conditions which once satisfied, lead to a positive conclusion terminating $[3,5,12,13,14,17,19,22,23$, $26,31]$, or on computing sufficient non-termination conditions which lead to a negative conclusion 
non-terminating [27, 28]. For convenience, we call the former computation a termination proof, and the latter a non-termination proof. Due to the nature of undecidability, there must be situations in which neither a termination proof nor a non-termination proof can apply; i.e., no sufficient termination/non-termination conditions are satisfied so that the user would get no conclusion (see the results of the Termination Competition 2007 [37]). We observe that in such a situation, it is particularly useful to compute an approximate conclusion indicating possible termination or possible non-termination, which guides the user to continue to improve his program towards termination. To the best of our knowledge, however, there is no existing approximation approach available. The goal of the current paper is then to develop such an approximation framework.

We propose an idea of termination prediction, as depicted in Figure 1. In the case that neither a termination nor a non-termination proof is applicable, we appeal to an approximation algorithm to predict possible termination or non-termination. The prediction applies to general logic programs with concrete or moded queries.

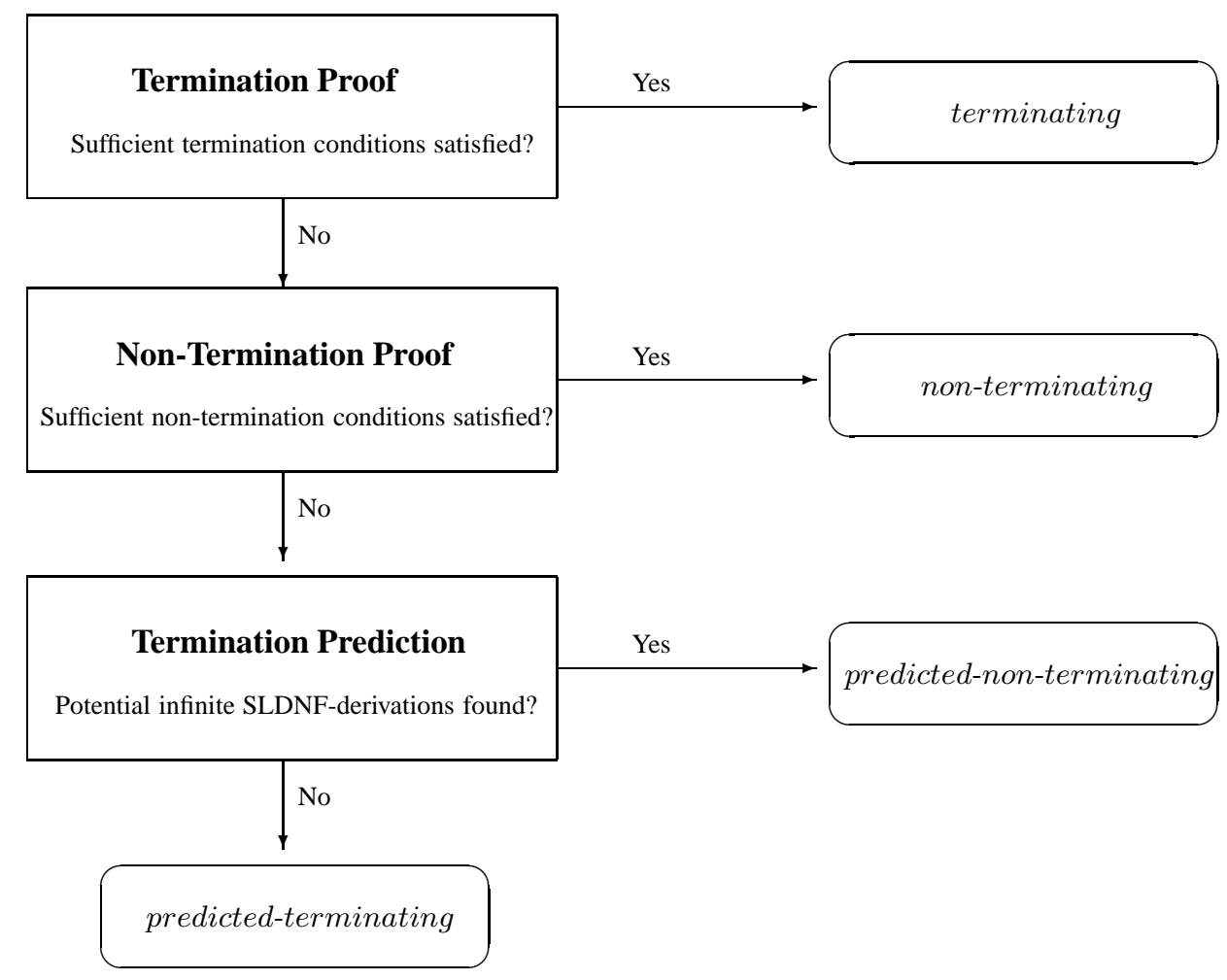

Figure 1: A framework for handling the termination problem

We develop a framework for predicting termination of general logic programs with arbitrary (i.e., concrete or moded) queries. The basic idea is that we establish a characterization of infinite (generalized) SLDNF-derivations with arbitrary queries. Then based on the characterization, we design a complete loop checking mechanism, which cuts all infinite SLDNF-derivations. Given a logic program and a query, we evaluate the query by applying SLDNF-resolution while performing 
loop checking. If the query evaluation proceeds without encountering potential infinite derivations, we predict terminating for this query; otherwise we predict non-terminating.

The core of our termination prediction is a characterization of infinite SLDNF-derivations with arbitrary queries. In [34], a characterization is established for general logic programs with concrete queries. This is far from enough for termination prediction; a characterization of infinite SLDNFderivations for moded queries is required. Moded queries are the most commonly used query form in static termination analysis. A moded query contains (abstract) atoms like $p(\mathcal{I}, T)$ where $T$ is a term (i.e., a constant, variable or function) and $\mathcal{I}$ is an input mode. An input mode stands for an arbitrary ground (i.e. variable-free) term, so that to prove that a logic program terminates for a moded query $p(\mathcal{I}, T)$ is to prove that the program terminates for any (concrete) query $p(t, T)$ where $t$ is a ground term.

It is nontrivial to characterize infinite SLDNF-derivations with moded queries. The first challenge we must address is how to formulate an SLDNF-derivation for a moded query $Q_{0}$, as the standard SLDNF-resolution is only for concrete queries $[9,18]$. We will introduce a framework called a moded-query forest, which consists of all (generalized) SLDNF-trees rooted at an instance of $Q_{0}$ (the instance is $Q_{0}$ with each input mode replaced by a ground term). An SLDNF-derivation for $Q_{0}$ is then defined over the moded-query forest such that a logic program $P$ terminates for $Q_{0}$ if and only if the moded-query forest contains no infinite SLDNF-derivations.

A moded-query forest may have an infinite number of SLDNF-trees, so it is infeasible for us to predict termination of a logic program by traversing the moded-query forest. To handle this challenge, we will introduce a novel compact approximation for a moded-query forest, called a moded generalized SLDNF-tree. The key idea is to treat an input mode as a special meta-variable in the way that during query evaluation, it can be substituted by a constant or function, but cannot be substituted by an ordinary variable. As a result, SLDNF-derivations for a moded query can be constructed in the same way as the ones for a concrete query. A characterization of infinite SLDNF-derivations for moded queries is then established in terms of some key properties of a moded generalized SLDNF-tree.

We have implemented a termination prediction tool and obtained quite satisfactory experimental results. Except for five programs which break the experiment time limit, our prediction is $100 \%$ correct for all benchmark programs of the Termination Competition 2007, of which eighteen programs cannot be proved by the existing state-of-the-art analyzers like AProVE07, NTI, Polytool and TALP.

The paper is organized as follows. Section 2 reviews some basic concepts including generalized SLDNF-trees. Sections 3 and 4 present a characterization of infinite SLDNF-derivations for concrete and moded queries, respectively. Section 5 introduces a new loop checking mechanism, and based on it develops an algorithm that predicts termination of general logic programs with arbitrary queries. The termination prediction method is illustrated with representative examples including ones borrowed from the Termination Competition 2007. Section 6 describes the im- 
plementation of our termination prediction algorithm and presents experimental results over the programs of the Termination Competition 2007. Section 7 mentions related work, and Section 8 concludes.

\section{Preliminaries}

We assume the reader is familiar with standard terminology of logic programs, in particular with SLDNF-resolution, as described in [18]. Variables begin with a capital letter $X, Y, Z, U, V$ or $I$, and predicate, function and constant symbols with a lower case letter. A term is a constant, a variable, or a function of the form $f\left(T_{1}, \ldots, T_{m}\right)$ where $f$ is a function symbol and each $T_{i}$ is a term. For simplicity, we use $\bar{T}$ to denote a set of terms $T_{1}, \ldots, T_{m}$. An atom is of the form $p(\bar{T})$ where $p$ is a predicate symbol. Let $A$ be an atom/term. The size of $A$, denoted $|A|$, is the number of occurrences of function symbols, variables and constants in $A$. Two atoms are called variants if they are the same up to variable renaming. A literal is an atom $A$ or the negation $\neg A$ of $A$.

A (general) logic program $P$ is a finite set of clauses of the form $A \leftarrow L_{1}, \ldots, L_{n}$, where $A$ is an atom and each $L_{i}$ is a literal. Throughout the paper, we consider only Herbrand models. The Herbrand universe and Herbrand base of $P$ are denoted by $H U(P)$ and $H B(P)$, respectively.

A goal $G_{i}$ is a headless clause $\leftarrow L_{1}, \ldots, L_{n}$ where each literal $L_{j}$ is called a subgoal. The goal, $G_{0}=\leftarrow Q_{0}$, for a query $Q_{0}$ is called a top goal. Without loss of generality, we assume that $Q_{0}$ consists only of one atom. $Q_{0}$ is a moded query if some arguments of $Q_{0}$ are input modes (in this case, $Q_{0}$ is called an abstract atom); otherwise, it is a concrete query. An input mode always begins with a letter $\mathcal{I}$.

Let $P$ be a logic program and $G_{0}$ a top goal. $G_{0}$ is evaluated by building a generalized SLDNFtree $G T_{G_{0}}$ as defined in [34], in which each node is represented by $N_{i}: G_{i}$ where $N_{i}$ is the name of the node and $G_{i}$ is a goal attached to the node. We do not reproduce the definition of a generalized SLDNF-tree. Roughly speaking, $G T_{G_{0}}$ is the set of standard SLDNF-trees for $P \cup\left\{G_{0}\right\}$ augmented with an ancestor-descendant relation on their subgoals. Let $L_{i}$ and $L_{j}$ be the selected subgoals at two nodes $N_{i}$ and $N_{j}$, respectively. $L_{i}$ is an ancestor of $L_{j}$, denoted $L_{i} \prec_{\text {anc }} L_{j}$, if the proof of $L_{i}$ goes via the proof of $L_{j}$. Throughout the paper, we choose to use the best-known depth-first, left-most control strategy, as is used in Prolog, to select nodes/goals and subgoals (it can be adapted to any other fixed control strategies). So by the selected subgoal in each node $N_{i}: \leftarrow L_{1}, \ldots, L_{n}$, we refer to the left-most subgoal $L_{1}$.

Recall that in SLDNF-resolution, let $L_{i}=\neg A$ be a ground negative subgoal selected at $N_{i}$, then (by the negation-as-failure rule [9]) a subsidiary child SLDNF-tree $T_{N_{i+1}: A}$ rooted at $N_{i+1}: \leftarrow A$ will be built to solve $A$. In a generalized SLDNF-tree $G T_{G_{0}}$, such parent and child SLDNF-trees are connected from $N_{i}$ to $N_{i+1}$ via a dotted edge “.. . " (called a negation arc), and $A$ at $N_{i+1}$ inherits all ancestors of $L_{i}$ at $N_{i}$. Therefore, a path of a generalized SLDNF-tree may come across several SLDNF-trees through dotted edges. Any such a path starting at the root node 
$N_{0}: G_{0}$ of $G T_{G_{0}}$ is called a generalized SLDNF-derivation.

We do not consider floundering queries; i.e., we assume that no non-ground negative subgoals are selected at any node of a generalized SLDNF-tree (see [34]).

Another feature of a generalized SLDNF-tree $G T_{G_{0}}$ is that each subsidiary child SLDNF-tree $T_{N_{i+1}: \leftarrow A}$ in $G T_{G_{0}}$ terminates (i.e. stops expanding its nodes) at the first success leaf. The intuition behind this is that it is absolutely unnecessary to exhaust the remaining branches because they would never generate any new answers for $A$ (since $A$ is ground). In fact, Prolog executes the same pruning by using a cut operator to skip the remaining branches once the first success leaf is generated (e.g. see SICStus Prolog [15]). To illustrate, consider the following logic program and top goal:

$$
\begin{array}{rlr}
P_{0}: & p \leftarrow \neg q . & C_{p_{1}} \\
& q . & C_{q_{1}} \\
& q \leftarrow q . & C_{q_{2}} \\
G_{0}: & \leftarrow p . &
\end{array}
$$

The generalized SLDNF-tree $G T_{G_{0}}$ for $P_{0} \cup\left\{G_{0}\right\}$ is depicted in Figure 2. Note that the subsidiary child SLDNF-tree $T_{N_{2}:-q}$ terminates at the first success leaf $N_{3}$, leaving $N_{4}$ not further expanded. As a result, all generalized SLDNF-derivations in $G T_{G_{0}}$ are finite.

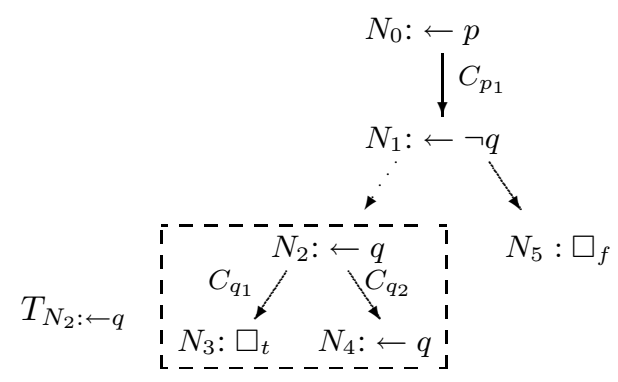

Figure 2: The generalized SLDNF-tree $G T_{G_{0}}$ of $P_{0}$.

For simplicity, in the following sections by a derivation or SLDNF-derivation we refer to a generalized SLDNF-derivation. Moreover, for any node $N_{i}: G_{i}$ we use $L_{i}^{1}$ to refer to the selected subgoal in $G_{i}$.

A derivation step is denoted by $N_{i}: G_{i} \Rightarrow_{C, \theta_{i}} N_{i+1}: G_{i+1}$, meaning that applying a clause $C$ to $G_{i}$ produces $N_{i+1}: G_{i+1}$, where $G_{i+1}$ is the resolvent of $C$ and $G_{i}$ on $L_{i}^{1}$ with the mgu (most general unifier) $\theta_{i}$. Here, for a substitution of two variables, $X$ in $L_{i}^{1}$ and $Y$ in (the head of) $C$, we always use $X$ to substitute for $Y$. When no confusion would occur, we may omit the mgu $\theta_{i}$ when writing a derivation step. 


\section{A Characterization of Infinite SLDNF-Derivations for Con- crete Queries}

In this section, we review the characterization of infinite derivations with concrete queries presented in [34].

Definition 1 Let $T$ be a term or an atom and $S$ be a string that consists of all predicate symbols, function symbols, constants and variables in $T$, which is obtained by reading these symbols sequentially from left to right. The symbol string of $T$, denoted $S_{T}$, is the string $S$ with every variable replaced by $\mathcal{X}$.

For instance, let $T_{1}=a$ and $T_{2}=f(X, g(X, f(a, Y)))$. Then $S_{T_{1}}=a$ and $S_{T_{2}}=f \mathcal{X} g \mathcal{X} f a \mathcal{X}$.

Definition 2 Let $S_{T_{1}}$ and $S_{T_{2}}$ be two symbol strings. $S_{T_{1}}$ is a projection of $S_{T_{2}}$, denoted $S_{T_{1}} \subseteq_{\text {proj }}$ $S_{T_{2}}$, if $S_{T_{1}}$ is obtained from $S_{T_{2}}$ by removing zero or more elements.

Definition 3 Let $A_{1}$ and $A_{2}$ be two atoms (positive subgoals) with the same predicate symbol. $A_{1}$ is said to loop into $A_{2}$, denoted $A_{1} \rightsquigarrow_{\text {loop }} A_{2}$, if $S_{A_{1}} \subseteq_{\text {proj }} S_{A_{2}}$. Let $N_{i}: G_{i}$ and $N_{j}: G_{j}$ be two nodes in a derivation with $L_{i}^{1} \prec_{\text {anc }} L_{j}^{1}$ and $L_{i}^{1} \rightsquigarrow l_{\text {loop }} L_{j}^{1}$. Then $G_{j}$ is called a loop goal of $G_{i}$.

Observe that if $A_{1} \rightsquigarrow_{\text {loop }} A_{2}$ then $\left|A_{1}\right| \leq\left|A_{2}\right|$, and that if $G_{3}$ is a loop goal of $G_{2}$ that is a loop goal of $G_{1}$ then $G_{3}$ is a loop goal of $G_{1}$. Since a logic program has only a finite number of clauses, an infinite derivation results from repeatedly applying the same set of clauses, which leads to either infinite repetition of selected variant subgoals or infinite repetition of selected subgoals with recursive increase in term size. By recursive increase of term size of a subgoal $A$ from a subgoal $B$ we mean that $A$ is $B$ with a few function/constant/variable symbols added and possibly with some variables changed to different variables. Such crucial dynamic characteristics of an infinite derivation are captured by loop goals. The following result is proved in [34].

Theorem 1 Let $G_{0}=\leftarrow Q_{0}$ be a top goal with $Q_{0}$ a concrete query. Any infinite derivation $D$ in $G T_{G_{0}}$ contains an infinite sequence of goals $G_{0}, \ldots, G_{g_{1}}, \ldots, G_{g_{2}}, \ldots$ such that for any $j \geq 1, G_{g_{j+1}}$ is a loop goal of $G_{g_{j}}$.

Put another way, Theorem 1 states that any infinite derivation $D$ in $G T_{G_{0}}$ is of the form

$$
N_{0}: G_{0} \Rightarrow_{C_{0}} \ldots N_{g_{1}}: G_{g_{1}} \Rightarrow_{C_{1}} \ldots N_{g_{2}}: G_{g_{2}} \Rightarrow_{C_{2}} \ldots N_{g_{3}}: G_{g_{3}} \Rightarrow_{C_{3}} \ldots
$$

where for any $j \geq 1, G_{g_{j+1}}$ is a loop goal of $G_{g_{j}}$. This provides a necessary and sufficient characterization of an infinite generalized SLDNF-derivation with a concrete query.

Example 1 Consider the following logic program: 


$$
\begin{array}{ll}
P_{1}: & p(a) . \\
& p(f(X)) \leftarrow p(X) .
\end{array}
$$

The generalized SLDNF-tree $G T_{\leftarrow p(X)}$ for a concrete query $p(X)$ is shown in Figure 3, where for simplicity the symbol $\leftarrow$ in each goal is omitted. Note that $G T_{\leftarrow p(X)}$ has an infinite derivation

$$
N_{0}: p(X) \Rightarrow_{C_{p_{2}}} N_{2}: p\left(X_{2}\right) \Rightarrow_{C_{p_{2}}} N_{4}: p\left(X_{4}\right) \Rightarrow_{C_{p_{2}}} \cdots
$$

where for any $j \geq 0, G_{2(j+1)}$ is a loop goal of $G_{2 j}$.

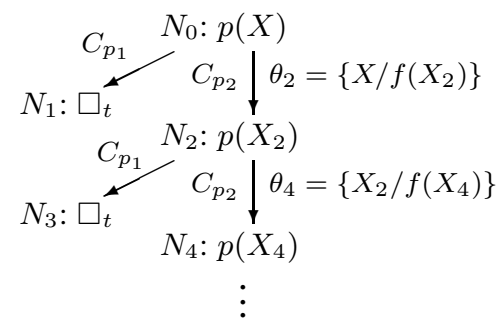

Figure 3: The generalized SLDNF-tree $G T_{\leftarrow p(X)}$ of $P_{1}$ for a concrete query $p(X)$.

\section{A Characterization of Infinite SLDNF-Derivations for Moded Queries}

We first define generalized SLDNF-derivations for moded queries by introducing a framework called moded-query forests.

Definition 4 Let $P$ be a logic program and $Q_{0}=p\left(\mathcal{I}_{1}, \ldots, \mathcal{I}_{m}, T_{1}, \ldots, T_{n}\right)$ a moded query. The moded-query forest of $P$ for $Q_{0}$, denoted $M F_{Q_{0}}$, consists of all generalized SLDNF-trees for $P \cup\left\{G_{0}\right\}$, where $G_{0}=\leftarrow p\left(t_{1}, \ldots, t_{m}, T_{1}, \ldots, T_{n}\right)$ with each $t_{i}$ being a ground term from $H U(P)$. A (generalized $S L D N F$-) derivation for the moded query $Q_{0}$ is a derivation in any generalized SLDNF-tree of $M F_{Q_{0}}$.

Therefore, a logic program $P$ terminates for a moded query $Q_{0}$ if and only if there is no infinite derivation for $Q_{0}$ if and only if $M F_{Q_{0}}$ has no infinite derivation.

Example 2 Consider the logic program $P_{1}$ again. We have $H U\left(P_{1}\right)=\{a, f(a), f(f(a)), \ldots\}$. Let $p(\mathcal{I})$ be a moded query. The moded-query forest $M F_{p(\mathcal{I})}$ consists of generalized SLDNFtrees $G T_{\leftarrow p(a)}, G T_{\leftarrow p(f(a))}$, etc., as shown in Figure 4. Note that $M F_{p(\mathcal{I})}$ has an infinite number of generalized SLDNF-trees. However, any individual tree, $G T_{G_{0}}$ with $\left.\left.G_{0}=\leftarrow p(\underbrace{f(f(\ldots f}_{n \text { items }}(a) \ldots)\right)\right)$ $(n \geq 0)$, is finite. $M F_{p(\mathcal{I})}$ contains no infinite derivation, thus $P_{1}$ terminates for $p(\mathcal{I})$. 


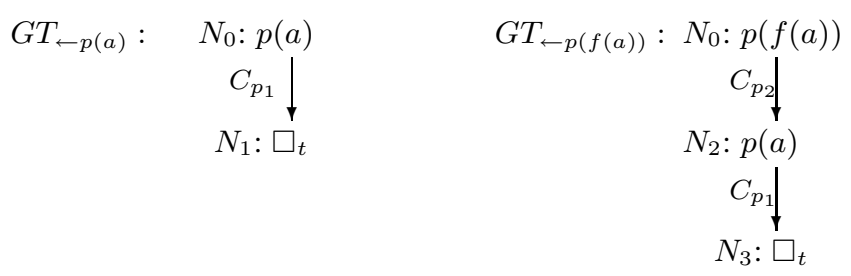

Figure 4: The moded-query forest $M F_{p(\mathcal{I})}$ of $P_{1}$ for a moded query $p(\mathcal{I})$.

In a moded-query forest, all input modes are instantiated into ground terms in $H U(P)$. When $H U(P)$ is infinite, the moded-query forest would contain infinitely many generalized SLDNFtrees. This means that it is infeasible to build a moded-query forest to represent the derivations for a moded query. An alternative yet ideal way is to directly apply SLDNF-resolution to evaluate input modes and build a compact generalized SLDNF-tree for a moded query. Unfortunately, SLDNF-resolution accepts only terms as arguments of a top goal; an input mode $\mathcal{I}$ is not directly evaluable.

Since an input mode stands for an arbitrary ground term, i.e. it can be any term from $H U(P)$, during query evaluation it can be instantiated to any term except variable (note that a ground term cannot be substituted by a variable). This suggests that we may approximate the effect of an input mode $\mathcal{I}$ by treating it as a special (meta-) variable $I$ in the way that in SLDNF-derivations, $I$ can be substituted by a constant or function, but cannot be substituted by an ordinary variable. Therefore, when doing unification of a special variable $I$ and a variable $X$, we always substitute $I$ for $X$.

Definition 5 Let $P$ be a logic program and $Q_{0}=p\left(\mathcal{I}_{1}, \ldots, \mathcal{I}_{m}, T_{1}, \ldots, T_{n}\right)$ a moded query. The moded generalized $S L D N F$-tree of $P$ for $Q_{0}$, denoted $M T_{Q_{0}}$, is defined to be the generalized SLDNF-tree $G T_{G_{0}}$ for $P \cup\left\{G_{0}\right\}$, where $G_{0}=\leftarrow p\left(I_{1}, \ldots, I_{m}, T_{1}, \ldots, T_{n}\right)$ with each $I_{i}$ being a distinct special variable not occurring in any $T_{j}$. The special variables $I_{1}, \ldots, I_{m}$ for the input modes $\mathcal{I}_{1}, \ldots, \mathcal{I}_{m}$ are called input mode variables (or input variables).

In a moded generalized SLDNF-tree, an input variable $I$ may be substituted by either a constant $t$ or a function $f(\bar{T})$. It will not be substituted by any non-input variable. If $I$ is substituted by $f(\bar{T})$, all variables in $\bar{T}$ are also called input variables (thus are treated as special variables).

In this paper, we do not consider floundering moded queries; i.e., we assume that no negative subgoals containing either ordinary or input variables are selected at any node of a moded generalized SLDNF-tree.

Definition 6 Let $P$ be a logic program, $Q_{0}=p\left(\mathcal{I}_{1}, \ldots, \mathcal{I}_{m}, T_{1}, \ldots, T_{n}\right)$ a moded query, and $G_{0}=\leftarrow$ $p\left(I_{1}, \ldots, I_{m}, T_{1}, \ldots, T_{n}\right)$. Let $D$ be a derivation in the moded generalized SLDNF-tree $M T_{Q_{0}}$. A moded instance of $D$ is a derivation obtained from $D$ by first instantiating all input variables at the root node $N_{0}: G_{0}$ with an mgu $\theta=\left\{I_{1} / t_{1}, \ldots, I_{m} / t_{m}\right\}$, where each $t_{i} \in H U(P)$, then passing the instantiation $\theta$ down to the other nodes of $D$. 
Example 3 Consider the logic program $P_{1}$ again. Let $Q_{0}=p(\mathcal{I})$ be a moded query and $G_{0}=\leftarrow$ $p(I)$. The moded generalized SLDNF-tree $M T_{Q_{0}}$ is $G T_{G_{0}}$ as depicted in Figure 5, where all input variables are underlined. Since $I$ is an input variable, $X_{2}$ is an input variable, too (due to the mgu $\left.\theta_{2}\right)$. For the same reason, all $X_{2 i}$ are input variables $(i>0)$.

Consider the following infinite derivation $D$ in $M T_{Q_{0}}$ :

$$
N_{0}: p(I) \Rightarrow_{C_{p_{2}}} N_{2}: p\left(X_{2}\right) \Rightarrow_{C_{p_{2}}} N_{4}: p\left(X_{4}\right) \Rightarrow_{C_{p_{2}}} \cdots
$$

By instantiating the input variable $I$ at $N_{0}$ with different ground terms from $H U\left(P_{1}\right)$ and passing the instantiation $\theta$ down to the other nodes of $D$, we can obtain different moded instances from $D$. For example, instantiating $I$ to $a$ (i.e. $\theta=\{I / a\}$ ) yields the moded instance

$$
N_{0}: p(a)
$$

Instantiating $I$ to $f(a)$ (i.e. $\theta=\{I / f(a)\}$ ) yields the moded instance

$$
N_{0}: p(f(a)) \Rightarrow_{C_{p_{2}}} N_{2}: p(a)
$$

And, instantiating $I$ to $f(f(a))$ (i.e. $\theta=\{I / f(f(a))\})$ yields the moded instance

$$
\begin{aligned}
& N_{0}: p(f(f(a))) \Rightarrow_{C_{p_{2}}} N_{2}: p(f(a)) \Rightarrow_{C_{p_{2}}} N_{4}: p(a) \\
& C_{N_{1}: \square_{t}}^{C_{p_{1}}} \overbrace{p_{p_{2}}: p(\underline{I})}^{N_{0}} \theta_{2}=\left\{\underline{I} / f\left(X_{2}\right)\right\} \\
& C_{p_{1}} N_{2}: p\left(\underline{X_{2}}\right) \\
& N_{3}: \square_{N_{4}: p\left(\underline{X_{4}}\right)}^{C_{p_{1}}} C_{p_{2}}^{N_{2}} \underline{\theta_{4}}=\left\{\underline{X_{2}} / f\left(X_{4}\right)\right\}
\end{aligned}
$$

Figure 5: The moded generalized SLDNF-tree $M T_{p(\mathcal{I})}$ of $P_{1}$ for a moded query $p(\mathcal{I})$.

Observe that a moded instance of a derivation $D$ in $M T_{Q_{0}}$ is a derivation in $G T_{G_{0} \theta}$, where $G_{0} \theta=\leftarrow p\left(t_{1}, \ldots, t_{m}, T_{1}, \ldots, T_{n}\right)$ with each $t_{i}$ being a ground term from $H U(P)$. By Definition 4 , $G T_{G_{0} \theta}$ is in the moded-query forest $M F_{Q_{0}}$. This means that any moded instance of a derivation in $M T_{Q_{0}}$ is a derivation for $Q_{0}$ in $M F_{Q_{0}}$. For instance, all moded instances illustrated in Example 3 are derivations in the moded-query forest $M F_{Q_{0}}$ of Figure 4.

Theorem 2 Let $M F_{Q_{0}}$ and $M T_{Q_{0}}$ be the moded-query forest and the moded generalized SLDNFtree of $P$ for $Q_{0}$, respectively. If $M F_{Q_{0}}$ has an infinite derivation $D^{\prime}, M T_{Q_{0}}$ has an infinite derivation $D$ with $D^{\prime}$ as a moded instance. 
Proof: Let $Q_{0}=p\left(\mathcal{I}_{1}, \ldots, \mathcal{I}_{m}, T_{1}, \ldots, T_{n}\right)$. Then, the root node of $D^{\prime}$ is $N_{0}: \leftarrow p\left(t_{1}, \ldots, t_{m}, T_{1}, \ldots, T_{n}\right)$ with each $t_{i} \in H U(P)$, and the root node of $M T_{Q_{0}}$ is $N_{0}: \leftarrow p\left(I_{1}, \ldots, I_{m}, T_{1}, \ldots, T_{n}\right)$ with each $I_{i}$ being an input variable not occurring in any $T_{j}$. Note that the former is an instance of the latter with the mgu $\theta=\left\{I_{1} / t_{1}, \ldots, I_{m} / t_{m}\right\}$. Let $D^{\prime}$ be of the form

$$
N_{0}: \leftarrow p\left(t_{1}, \ldots, t_{m}, T_{1}, \ldots, T_{n}\right) \Rightarrow_{C_{0}} N_{1}: G_{1}^{\prime} \cdots \Rightarrow_{C_{i}} N_{i+1}: G_{i+1}^{\prime} \cdots
$$

$M T_{Q_{0}}$ must have a derivation $D$ of the form

$$
N_{0}: \leftarrow p\left(I_{1}, \ldots, I_{m}, T_{1}, \ldots, T_{n}\right) \Rightarrow_{C_{0}} N_{1}: G_{1} \cdots \Rightarrow_{C_{i}} N_{i+1}: G_{i+1} \cdots
$$

such that each $G_{i}^{\prime}=G_{i} \theta$, since for any $i \geq 0$ and any clause $C_{i}$ in $P$, if $G_{i}^{\prime}$ can unify with $C_{i}$, so can $G_{i}$ with $C_{i}$. Note that when the selected subgoal at some $G_{i}^{\prime}$ is a negative ground literal, by the assumption that $Q_{0}$ is non-floundering, we have the same selected literal at $G_{i}$. We then have the proof.

Our goal is to establish a characterization of infinite derivations for a moded query such that the converse of Theorem 2 is true under some conditions.

Consider the infinite derivation in Figure 5 again. The input variable $I$ is substituted by $f\left(X_{2}\right)$; $X_{2}$ is then substituted by $f\left(X_{4}\right), \ldots$ This produces an infinite chain of substitutions for $I$ of the form $I / f\left(X_{2}\right), X_{2} / f\left(X_{4}\right), \ldots$ The following lemma shows that infinite derivations containing such an infinite chain of substitutions have no infinite moded instances.

Lemma 1 If a derivation $D$ in a moded generalized SLDNF-tree $M T_{Q_{0}}$ is infinite but none of its moded instances is infinite, then there is an input variable I such that D contains an infinite chain of substitutions for I of the form

$$
I / f_{1}\left(\ldots, Y_{1}, \ldots\right), \ldots, Y_{1} / f_{2}\left(\ldots, Y_{2}, \ldots\right), \ldots, Y_{i-1} / f_{i}\left(\ldots, Y_{i}, \ldots\right), \ldots
$$

(some $f_{i}$ s would be the same).

Proof: We distinguish four types of substitution chains for an input variable $I$ in $D$ :

1. $X_{1} / I, \ldots, X_{m} / I$ or $X_{1} / I, \ldots, X_{i} / I, \ldots$ That is, $I$ is never substituted by any terms.

2. $X_{1} / I, \ldots, X_{m} / I, I / t$ where $t$ is a ground term. That is, $I$ is substituted by a ground term.

3. $X_{1} / I, \ldots, X_{m} / I, I / f_{1}\left(\ldots, Y_{1}, \ldots\right), \ldots, Y_{1} / f_{2}\left(\ldots, Y_{2}, \ldots\right), \ldots, Y_{n-1} / f_{n}\left(\ldots, Y_{n}, \ldots\right), \ldots$, where $f_{n}($ $\left.\ldots, Y_{n}, \ldots\right)$ is the last non-ground function in the substitution chain for $I$ in $D$. In this case, $I$ is recursively substituted by a finite number of functions.

4. $X_{1} / I, \ldots, X_{m} / I, I / f_{1}\left(\ldots, Y_{1}, \ldots\right), \ldots, Y_{1} / f_{2}\left(\ldots, Y_{2}, \ldots\right), \ldots, Y_{i-1} / f_{i}\left(\ldots, Y_{i}, \ldots\right), \ldots$ In this case, $I$ is recursively substituted by an infinite number of functions. 
For type 1, $D$ retains its infinite extension for whatever ground term we replace $I$ with. For type $2, D$ retains its infinite extension when we use $t$ to replace $I$. To sum up, for any input variable $I$ whose substitution chain is of type 1 or of type 2 , there is a ground term $t$ such that replacing $I$ with $t$ does not affect the infinite extension of $D$. In this case, replacing $I$ in $D$ with $t$ leads to an infinite derivation less general than $D$.

For type 3 , note that all variables appearing in the $f_{i}($.$) s are input variables. Since f_{n}\left(\ldots, Y_{n}, \ldots\right)$ is the last non-ground function in the substitution chain for $I$ in $D$, the substitution chain for every variable $Y_{n}$ in $f_{n}\left(\ldots, Y_{n}, \ldots\right)$ is either of type 1 or of type 2 . Therefore, we can replace each $Y_{n}$ with an appropriate ground term $t_{n}$ without affecting the infinite extension of $D$. After this replacement, $D$ becomes $D_{n}$ and $f_{n}\left(\ldots, Y_{n}, \ldots\right)$ becomes a ground term $f_{n}\left(\ldots, t_{n}, \ldots\right)$. Now $f_{n-1}\left(\ldots, Y_{n-1}, \ldots\right)$ is the last non-ground function in the substitution chain for $I$ in $D_{n}$. Repeating the above replacement recursively, we will obtain an infinite derivation $D_{1}$, which is $D$ with all variables in the $f_{i}($.$) s replaced with a ground term. Assume f_{1}\left(\ldots, Y_{1}, \ldots\right)$ becomes a ground term $t$ in $D_{1}$. Then the substitution chain for $I$ in $D_{1}$ is of type 2. So replacing $I$ with $t$ in $D_{1}$ leads to an infinite derivation $D_{0}$.

The above constructive proof shows that if the substitution chains for all input variables in $D$ are of type 1, 2 or 3, then $D$ must have an infinite moded instance. Since $D$ has no infinite moded instance, there must exist an input variable $I$ whose substitution chain in $D$ is of type 4 . That is, $I$ is recursively substituted by an infinite number of functions. Note that some $f_{i}$ s would be the same because a logic program has only a finite number of function symbols. This concludes the proof.

We are ready to introduce the following principal result.

Theorem 3 Let $M F_{Q_{0}}$ and $M T_{Q_{0}}$ be the moded-query forest and the moded generalized SLDNFtree of $P$ for $Q_{0}$, respectively. $M F_{Q_{0}}$ has an infinite derivation if and only if $M T_{Q_{0}}$ has an infinite derivation $D$ of the form

$$
N_{0}: G_{0} \Rightarrow_{C_{0}} \ldots N_{g_{1}}: G_{g_{1}} \Rightarrow_{C_{1}} \ldots N_{g_{2}}: G_{g_{2}} \Rightarrow_{C_{2}} \ldots N_{g_{3}}: G_{g_{3}} \Rightarrow_{C_{3}} \ldots
$$

where ( $i$ ) for any $j \geq 1, G_{g_{j+1}}$ is a loop goal of $G_{g_{j}}$, and (ii) for no input variable $I$, $D$ contains an infinite chain of substitutions for I of form (1).

Proof: $(\Longrightarrow)$ Assume $M F_{Q_{0}}$ has an infinite derivation $D^{\prime}$. By Theorem 2, $G T_{G_{0}}$ has an infinite derivation $D$ with $D^{\prime}$ as a moded instance. By Theorem 1, D is of form (2) and satisfies condition (i).

Assume, on the contrary, that $D$ does not satisfy condition (ii). That is, for some input variable $I, D$ contains an infinite chain of substitutions for $I$ of the form

$$
I / f_{1}\left(\ldots, Y_{1}, \ldots\right), \ldots, Y_{1} / f_{2}\left(\ldots, Y_{2}, \ldots\right), \ldots, Y_{i-1} / f_{i}\left(\ldots, Y_{i}, \ldots\right), \ldots
$$


Note that for whatever ground term $t$ we assign to $I$, this chain can be instantiated at most as long in length as the following one:

$$
t / f_{1}\left(\ldots, t_{1}, \ldots\right), \ldots, t_{1} / f_{2}\left(\ldots, t_{2}, \ldots\right), \ldots, t_{k} / f_{k+1}\left(\ldots, Y_{k+1}, \ldots\right)
$$

where $k=|t|, t_{i}$ s are ground terms and $\left|t_{k}\right|=1$. This means that replacing $I$ with any ground term $t$ leads to a finite moded instance of $D$. Therefore, $D$ has no infinite moded instance in $M F_{Q_{0}}$, a contradiction.

$(\Longleftarrow)$ Assume, on the contrary, that $M F_{Q_{0}}$ has no infinite derivation. By Lemma 1, we reach a contradiction to condition (ii).

Theorem 3 provides a necessary and sufficient characterization of an infinite generalized SLDNF-derivation for a moded query. Note that it coincides with Theorem 1 when $Q_{0}$ is a concrete query, where $M F_{Q_{0}}=M T_{Q_{0}}$ and condition (ii) is always true.

The following corollary is immediate to this theorem.

Corollary 1 A logic program $P$ terminates for a moded query $Q_{0}$ if and only if the moded generalized SLDNF-tree $M T_{Q_{0}}$ has no infinite derivation of form (2) satisfying conditions (i) and (ii) of Theorem 3.

We use simple yet typical examples to illustrate the proposed characterization of infinite SLDNF-derivations with moded queries.

Example 4 Consider the moded generalized SLDNF-tree $M T_{Q_{0}}$ in Figure 5. It has only one infinite derivation, which satisfies condition (i) of Theorem 3 where for each $j \geq 0, N_{g_{j}}$ in Theorem 3 corresponds to $N_{2 j}$ in Figure 5. However, the chain of substitutions for $I$ in this derivation violates condition (ii). This means that $M F_{Q_{0}}$ contains no infinite derivations; therefore, there is no infinite derivation for the moded query $p(\mathcal{I})$. As a result, $P_{1}$ terminates for $p(\mathcal{I})$.

Example 5 Consider the following logic program:

$$
\begin{array}{lll}
P_{2}: & \operatorname{append}([], X, X) . & C_{a_{1}} \\
& \operatorname{append}([X \mid Y], U,[X \mid Z]) \leftarrow \operatorname{append}(Y, U, Z) . & C_{a_{2}}
\end{array}
$$

Let us choose the three simplest moded queries:

$$
\begin{aligned}
& Q_{0}^{1}=\operatorname{append}\left(\mathcal{I}, V_{2}, V_{3}\right), \\
& Q_{0}^{2}=\operatorname{append}\left(V_{1}, \mathcal{I}, V_{3}\right), \\
& Q_{0}^{3}=\operatorname{append}\left(V_{1}, V_{2}, \mathcal{I}\right) .
\end{aligned}
$$

Since applying clause $C_{a_{1}}$ produces only leaf nodes, for simplicity we ignore it when depicting moded generalized SLDNF-trees. The three moded generalized SLDNF-trees $M T_{Q_{0}^{1}}, M T_{Q_{0}^{2}}$ and $M T_{Q_{0}^{3}}$ are shown in Figures 6 (a), (b) and (c), respectively. Note that all the derivations are infinite 
and satisfy condition (i) of Theorem 3, where for each $j \geq 0, N_{g_{j}}$ in Theorem 3 corresponds to $N_{j}$ in Figure 6. Apparently, the chains of substitutions for $I$ in the derivations of $M T_{Q_{0}^{1}}$ and $M T_{Q_{0}^{3}}$ violate condition (ii) of Theorem 3. $M F_{Q_{0}^{1}}$ and $M F_{Q_{0}^{3}}$ contain no infinite derivation and thus there exists no infinite derivation for the moded queries $Q_{0}^{1}$ and $Q_{0}^{3}$. Therefore, $P_{2}$ terminates for $Q_{0}^{1}$ and $Q_{0}^{3}$. However, the derivation in $M T_{Q_{0}^{2}}$ satisfies condition (ii), thus there exist infinite derivations for the moded query $Q_{0}^{2}$. $P_{2}$ does not terminate for $Q_{0}^{2}$.

$$
\begin{gathered}
N_{0}: \text { append }\left(\underline{I}, V_{2}, V_{3}\right) \\
C_{a_{2}} \mid \begin{array}{l}
\theta_{0}=\{\underline{I} /[X \mid Y], \\
\left.U / V_{2}, V_{3} /[X \mid Z]\right\}
\end{array} \\
N_{1}: \text { append }\left(\underline{Y}, V_{2}, Z\right) \\
C_{a_{2}} \mid \begin{array}{l}
\theta_{1}=\left\{\underline{Y} /\left[X_{1} \mid Y_{1}\right],\right. \\
\left.U_{1} / V_{2}, Z /\left[X_{1} \mid Z_{1}\right]\right\}
\end{array} \\
N_{2}: \operatorname{append}\left(\underline{Y_{1}}, V_{2}, Z_{1}\right)
\end{gathered}
$$

(a)

$$
\begin{gathered}
N_{0}: \operatorname{append}\left(V_{1}, \underline{I}, V_{3}\right) \\
C_{a_{2}} \mid \begin{array}{l}
\theta_{0}=\left\{V_{1} /[X \mid Y],\right. \\
\left.U / \underline{I}, V_{3} /[X \mid Z]\right\}
\end{array} \\
N_{1}: \operatorname{append}(Y, \underline{I}, Z) \\
C_{a_{2}} \mid \begin{array}{l}
\theta_{1}=\left\{Y /\left[X_{1} \mid Y_{1}\right],\right. \\
\left.U_{1} / \underline{I}, Z /\left[X_{1} \mid Z_{1}\right]\right\}
\end{array} \\
N_{2}: \operatorname{append}\left(Y_{1}, \underline{I}, Z_{1}\right)
\end{gathered}
$$

(b)

$$
\begin{gathered}
N_{0}: \operatorname{append}\left(V_{1}, V_{2}, \underline{I}\right) \\
C_{a_{2}} \mid \begin{array}{l}
\theta_{0}=\left\{V_{1} /[X \mid Y],\right. \\
\left.U / V_{2}, \underline{I} /[X \mid Z]\right\}
\end{array} \\
N_{1}: \operatorname{append}\left(Y, V_{2}, \underline{Z}\right) \\
C_{a_{2}} \mid \begin{array}{l}
\theta_{1}=\left\{Y /\left[X_{1} \mid Y_{1}\right],\right. \\
\left.U_{1} / V_{2}, \underline{Z} /\left[X_{1} \mid Z_{1}\right]\right\}
\end{array} \\
N_{2}: \operatorname{append}\left(Y_{1}, V_{2}, \underline{Z_{1}}\right)
\end{gathered}
$$

(c)

Figure 6: (a) $M T_{Q_{0}^{1}}$, (b) $M T_{Q_{0}^{2}}$, and (c) $M T_{Q_{0}^{3}}$.

Let $\operatorname{pred}(P)$ be the set of predicate symbols in $P$. Define

$$
\begin{aligned}
M Q(P) & =\left\{p\left(\mathcal{I}_{1}, \ldots, \mathcal{I}_{m}, X_{m+1}, \ldots, X_{n}\right) \mid p\right. \text { is an } \\
& n \text {-ary predicate symbol in } \operatorname{pred}(P) \text { and } m>0\} .
\end{aligned}
$$

Note that $M Q(P)$ contains all most general moded queries of $P$ in the sense that any moded query of $P$ is an instance of some query in $M Q(P)$. Since $\operatorname{pred}(P)$ is finite, $M Q(P)$ is finite. Therefore, it is immediate that $P$ terminates for all moded queries if and only if it terminates for each moded query in $M Q(P)$.

Theorem 4 For any two moded queries $Q_{0}^{1}=p\left(\mathcal{I}_{1}, \ldots, \mathcal{I}_{l}, X_{l+1}, \ldots, X_{n}\right)$ and $Q_{0}^{2}=p\left(\mathcal{I}_{1}, \ldots, \mathcal{I}_{m}\right.$, $\left.X_{m+1}, \ldots, X_{n}\right)$ with $l<m$, that there is no infinite derivation for $Q_{0}^{1}$ implies there is no infinite derivation for $Q_{0}^{2}$.

Proof: Note that we consider only non-floundering queries. Then, for any concrete query $Q$, that there is no infinite derivation for $Q$ implies there is no infinite derivation for any instance of $Q$. Assume that there is no infinite derivation for $Q_{0}^{1}$. Then, there is no infinite derivation for any query $Q=p\left(t_{1}, \ldots, t_{l}, X_{l+1}, \ldots, X_{n}\right)$, where each $t_{i}$ is a ground term from $H U(P)$. Then, there is no infinite derivation for any query $Q^{\prime}=p\left(t_{1}, \ldots, t_{l}, s_{l+1}, \ldots, s_{m}, X_{m+1}, \ldots, X_{n}\right)$, where each $t_{i}$ is a ground term from $H U(P)$ and each $s_{i}$ an instance of $X_{i}$. Since all $X_{i}$ s are variables, there is no infinite derivation for any query $Q^{\prime \prime}=p\left(t_{1}, \ldots, t_{l}, t_{l+1}, \ldots, t_{m}, X_{m+1}, \ldots, X_{n}\right)$, where each $t_{i}$ is a ground term from $H U(P)$. That is, there is no infinite derivation for $Q_{0}^{2}$.

Applying this theorem, we can conclude that $P_{2}$ in Example 5 terminates for all moded queries in $M Q\left(P_{2}\right)$ except $Q_{0}^{2}$. 


\section{An Algorithm for Predicting Termination of Logic Programs}

We develop an algorithm for predicting termination of logic programs based on the necessary and sufficient characterization of an infinite generalized SLDNF-derivation (Theorem 3 and Corollary 1). We begin by introducing a loop checking mechanism.

A loop checking mechanism, or more formally a loop check [4], defines conditions for us to cut a possibly infinite derivation at some node. By cutting a derivation at a node $N$ we mean removing all descendants of $N$. Informally, a loop check is said to be weakly sound if for any generalized SLDNF-tree $G T_{G_{0}}, G T_{G_{0}}$ having a success derivation before cut implies it has a success derivation after cut; it is said to be complete if it cuts all infinite derivations in $G T_{G_{0}}$. An ideal loop check cuts all infinite derivations while retaining success derivations. Unfortunately, as shown by Bol et al. [4], there exists no loop check that is both weakly sound and complete. In this paper, we focus on complete loop checks, because we want to apply them to predict termination of logic programs.

Definition 7 Given a repetition number $r \geq 2, L P$-check is defined as follows: Any derivation $D$ in a generalized SLDNF-tree is cut at a node $N_{g_{r}}$ if $D$ has a prefix of the form

$$
N_{0}: G_{0} \Rightarrow_{C_{0}} \ldots N_{g_{1}}: G_{g_{1}} \Rightarrow_{C_{k}} \ldots N_{g_{2}}: G_{g_{2}} \Rightarrow_{C_{k}} \ldots N_{g_{r}}: G_{g_{r}} \Rightarrow_{C_{k}}
$$

such that (a) for any $j<r, G_{g_{j+1}}$ is a loop goal of $G_{g_{j}}$, and (b) for all $j \leq r$, the clause $C_{k}$ applied to $G_{g_{j}}$ is the same. $C_{k}$ is then called a looping clause.

LP-check predicts infinite derivations from prefixes of derivations based on the characterization of Theorem 1 (or condition (i) of Theorem 3). The repetition number $r$ specifies the minimum number of loop goals appearing in the prefixes. It appears not appropriate to choose $r<2$, as that may lead to many finite derivations being wrongly cut. Although there is no mathematical mechanism available for choosing this repetition number (since the termination problem is undecidable), in many situations it suffices to choose $r=3$ for a correct prediction of infinite derivations. For instance, choosing $r=3$ we are able to obtain correct predictions for all benchmark programs of the Termination Competition 2007 (see Section 6).

LP-check applies to any generalized SLDNF-trees including moded generalized SLDNF-trees.

Theorem 5 LP-check is a complete loop check.

Proof: Let $D$ be an infinite derivation in $G T_{G_{0}}$. By Theorem 1, $D$ is of the form

$$
N_{0}: G_{0} \Rightarrow_{C_{0}} \ldots N_{f_{1}}: G_{f_{1}} \Rightarrow_{C_{1}} \ldots N_{f_{2}}: G_{f_{2}} \Rightarrow_{C_{2}} \ldots
$$

such that for any $i \geq 1, G_{f_{i+1}}$ is a loop goal of $G_{f_{i}}$. Since a logic program has only a finite number of clauses, there must be a (looping) clause $C_{k}$ being repeatedly applied at infinitely many nodes $N_{g_{1}}: G_{g_{1}}, N_{g_{2}}: G_{g_{2}}, \cdots$ where for each $j \geq 1, g_{j} \in\left\{f_{1}, f_{2}, \ldots\right\}$. Then for any $r>0, D$ has a

partial derivation of form (3). So $D$ will be cut at node $N_{g_{r}}: G_{g_{r}}$. This shows that any infinite derivation can be cut by LP-check. That is, LP-check is a complete loop check. 
Example 6 Let us choose $r=3$ and consider the infinite derivation $D$ in Figure 5. $p\left(X_{4}\right)$ at $N_{4}$ is a loop goal of $p\left(X_{2}\right)$ at $N_{2}$ that is a loop goal of $p(I)$ at $N_{0}$. Moreover, the same clause $C_{p_{2}}$ is applied at the three nodes. $D$ satisfies the conditions of LP-check and is cut at node $N_{4}$.

Recall that to prove that a logic program $P$ terminates for a moded query $Q_{0}=p\left(\mathcal{I}_{1}, \ldots, \mathcal{I}_{m}\right.$, $\left.T_{1}, \ldots, T_{n}\right)$ is to prove that $P$ terminates for any query $p\left(t_{1}, \ldots, t_{m}, T_{2}, \ldots, T_{n}\right)$, where each $t_{i}$ is a ground term. This can be reformulated in terms of a moded-query forest; that is, $P$ terminates for $Q_{0}$ if and only if $M F_{Q_{0}}$ has no infinite derivation. Then, Corollary 1 shows that $P$ terminates for $Q_{0}$ if and only if the moded generalized SLDNF-tree $M T_{Q_{0}}$ has no infinite derivation $D$ of form (2) satisfying the two conditions (i) and (ii) of Theorem 3. Although this characterization cannot be directly used for automated termination test because it requires generating infinite derivations in $M T_{Q_{0}}$, it can be used along with LP-check to predict termination, as LP-check is able to guess if a partial derivation would extend to an infinite one. Before describing our prediction algorithm with this idea, we introduce one more condition following Definition 7.

Definition 8 Let $D$ be a prefix of form (3). $D$ is said to have the term-size decrease property if for any $i$ with $0<i<r$, there is a substitution $X / f(\ldots Y \ldots)$ between $N_{g_{i}}$ and $N_{g_{i+1}}$, where $X$ is an input variable and $Y$ (an ordinary or input variable) appears in the selected subgoal of $G_{g_{i+1}}$.

Theorem 6 Let $D$ be a prefix of form (3) with $r \rightarrow \infty$. If $D$ has the term-size decrease property then $D$ contains an infinite chain of substitutions of form (1) for some input variable I at the root node of $D$.

Proof: First note that when $r \rightarrow \infty$ and $D$ has the term-size decrease property, $D$ contains an infinite number of substitutions of the form $X / f(\ldots)$, where $X$ is an input variable. Assume, on the contrary, that $D$ does not contain such an infinite chain of form (1). Let $M$ be the longest length of substitutions of form (1) for each input variable $I$ at the root node of $D$. Note that each input variable can be substituted only by a constant or function. For each substitution $X / f(\ldots)$ with $X$ an input variable, assume $f(\ldots)$ contains at most $N$ variables (i.e., it introduces at most $N$ new input variables). Then, $D$ contains at most $K *\left(N^{0}+N^{1}+\ldots+N^{M}\right)$ substitutions of the form $X / f(\ldots)$, where $K$ is the number of input variables at the root node of $D$ and $X$ is an input variable. This contradicts the condition that $D$ contains an infinite number of such substitutions. We conclude the proof.

LP-check and the term-size decrease property approximate conditions (i) and (ii) of Theorem 3 , respectively. So, we can guess an infinite extension (2) from a prefix (3) by combining the two mechanisms, as described in the following algorithm.

Algorithm 1 Predicting termination of a $\operatorname{logic}$ program $P$ for a (concrete or moded) query $Q_{0}$, given a repetition number $r>1(r=3$ is recommended). 
1. Initially, set $L=0$. Construct the moded generalized SLDNF-tree $M T_{Q_{0}}$ of $P$ for $Q_{0}$ in the way that whenever a prefix $D$ of the form

$$
N_{0}: G_{0} \Rightarrow_{C_{0}} \ldots N_{g_{1}}: G_{g_{1}} \Rightarrow_{C_{k}} \ldots N_{g_{2}}: G_{g_{2}} \Rightarrow_{C_{k}} \ldots N_{g_{r}}: G_{g_{r}} \Rightarrow_{C_{k}}
$$

is produced which satisfies conditions (a) and (b) of LP-check, if $D$ does not have the termsize decrease property then goto 3; else set $L=1$ and extend $D$ from the node $N_{g_{r}}$ with the looping clause $C_{k}$ skipped.

2. Return terminating if $L=0$; otherwise, return predicted-terminating.

3. Return predicted-non-terminating.

Starting from the root node $N_{0}: G_{0}$, we generate derivations of a moded generalized SLDNFtree $M T_{Q_{0}}$ step by step. If a prefix $D$ of form (3) is generated which satisfies conditions (a) and (b) of LP-check, then by Theorem $1 D$ is very likely to extend infinitely in $M T_{Q_{0}}$ (via the looping clause $C_{k}$ ). By Theorem 2, however, $D$ may not have infinite moded instances in $M F_{Q_{0}}$. So in this case, we further check if $D$ has the term-size decrease property. If not, by Theorem $3 D$ is very likely to have moded instances that extend infinitely in $M F_{Q_{0}}$. Algorithm 1 then predicts non-terminating for $Q_{0}$ by returning an answer predicted-non-terminating. If $D$ has the term-size decrease property, however, we continue to extend $D$ from $N_{g_{r}}$ by skipping the clause $C_{k}$ (i.e., the derivation via $C_{k}$ is cut at $N_{g_{r}}$ by LP-check).

When the answer is not predicted-non-terminating, we distinguish between two cases: (1) $L=$ 0 . This shows that no derivation was cut by LP-check during the construction of $M T_{Q_{0}}$. Algorithm 1 concludes terminating for $Q_{0}$ by returning an answer terminating. (2) $L=1$. This means that some derivations were cut by LP-check, all of which have the term-size decrease property. Algorithm 1 then predicts terminating for $Q_{0}$ by returning an answer predicted-terminating.

Note that for a concrete query $Q_{0}$, no derivation has the term-size decrease property. Therefore, Algorithm 1 returns predicted-non-terminating for $Q_{0}$ once a prefix of a derivation satisfying the conditions of LP-check is generated.

Theorem 7 For any logic program $P$, concrete/moded query $Q_{0}$ and repetition number $r$, Algorithm 1 always terminates.

Proof: Algorithm 1 constructs $M T_{Q_{0}}$ while applying LP-check to cut possible infinite derivations. Since LP-check is a complete loop check, it cuts all infinite derivations at some depth. This means that $M T_{Q_{0}}$ after cut by LP-check is finite. So, Algorithm 1 always terminates.

Algorithm 1 yields an approximate answer, predicted-terminating or predicted-non-terminating, or an exact answer terminating, as shown by the following theorem.

Theorem 8 P terminates for $Q_{0}$ if Algorithm 1 returns terminating. 
Proof: If Algorithm 1 returns terminating, no derivations were cut by LP-check; so the moded generalized SLDNF-tree $M T_{Q_{0}}$ for $Q_{0}$ is finite. By Corollary $1, P$ terminates for $Q_{0}$.

In the following examples, we choose a repetition number $r=3$.

Example 7 Consider Figure 5. Since the prefix $D$ between $N_{0}$ and $N_{4}$ satisfies the conditions of LP-check, Algorithm 1 concludes that the derivation may extend infinitely in $M T_{Q_{0}}$. It then checks the term-size decrease property to see if $D$ has moded instances that would extend infinitely in $M F_{Q_{0}}$. Clearly, $D$ has the term-size decrease property. So Algorithm 1 skips $C_{p_{2}}$ at $N_{4}$ (the branch is cut by LP-check). Consequently, Algorithm 1 predicts terminating for $p(\mathcal{I})$ by returning an answer predicted-terminating. This prediction is correct; see Example 4.

Example 8 Consider Figure 6. All the derivations starting at $N_{0}$ and ending at $N_{2}$ satisfy the conditions of LP-check, so they are cut at $N_{2}$. Since the derivations in $M T_{Q_{0}^{1}}$ and $M T_{Q_{0}^{3}}$ have the term-size decrease property, Algorithm 1 returns predicted-terminating for $Q_{0}^{1}$ and $Q_{0}^{3}$. Since the derivation in $M T_{Q_{0}^{2}}$ does not have the term-size decrease property, Algorithm 1 returns predictednon-terminating for $Q_{0}^{2}$. These predictions are all correct; see Example 5.

Example 9 Consider the following logic program $P_{3}$ :

$$
\begin{array}{lc}
\operatorname{mult}(s(X), Y, Z) \leftarrow \operatorname{mult}(X, Y, U), \operatorname{add}(U, Y, Z) . & C_{m_{1}} \\
\operatorname{mult}(0, Y, 0) . & C_{m_{2}} \\
\operatorname{add}(s(X), Y, s(Z)) \leftarrow \operatorname{add}(X, Y, Z) . & C_{a_{1}} \\
\operatorname{add}(0, Y, Y) . & C_{a_{2}}
\end{array}
$$

$M Q\left(P_{3}\right)$ consists of fourteen moded queries, seven for predicate mult and seven for predicate $a d d$. Applying Algorithm 1 yields the following result: (1) $P_{3}$ is predicted-terminating for all moded queries to add except add $\left(V_{1}, \mathcal{I}_{2}, V_{3}\right)$ for which $P_{3}$ is predicted-non-terminating, and (2) $P_{3}$ is predicted-terminating for $\operatorname{mult}\left(\mathcal{I}_{1}, \mathcal{I}_{2}, V_{3}\right)$ and mult $\left(\mathcal{I}_{1}, \mathcal{I}_{2}, \mathcal{I}_{3}\right)$, but is predicted-non-terminating for the remaining moded queries to mult. For illustration, we depict two moded generalized $\operatorname{SLDNF}$-trees for $\operatorname{mult}\left(\mathcal{I}, V_{2}, V_{3}\right)$ and $\operatorname{mult}\left(\mathcal{I}_{1}, \mathcal{I}_{2}, V_{3}\right)$, as shown in Figures 7 (a) and (b), respectively. In the two moded generalized SLDNF-trees, the prefix from $N_{0}$ down to $N_{2}$ satisfies the conditions of LP-check and has the term-size decrease property, so clause $C_{m_{1}}$ is skipped when expanding $N_{2}$. When the derivation is extended to $N_{6}$, the conditions of LP-check are satisfied again, where $G_{6}$ is a loop goal of $G_{5}$ that is a loop goal of $G_{4}$. Since the derivation for $\operatorname{mult}\left(\mathcal{I}, V_{2}, V_{3}\right)$ (Figure 7 (a)) does not have the term-size decrease property, Algorithm 1 returns an answer, predicted-non-terminating, for this moded query. The derivation for $\operatorname{mult}\left(\mathcal{I}_{1}, \mathcal{I}_{2}, V_{3}\right)$ (Figure 7 (b)) has the term-size decrease property, so clause $C_{a_{1}}$ is skipped when expanding $N_{6}$. For simplicity, we omitted all derivations leading to a success leaf. Because all derivations satisfying the conditions of LP-check have the term-size decrease property, Algorithm 1 ends with an answer, predicted-terminating, for $\operatorname{mult}\left(\mathcal{I}_{1}, \mathcal{I}_{2}, V_{3}\right)$. It is then immediately inferred by Theorem 
4 that $P_{3}$ is predicted-terminating for $\operatorname{mult}\left(\mathcal{I}_{1}, \mathcal{I}_{2}, \mathcal{I}_{3}\right)$. It is not difficult to verify that all these predictions are correct.

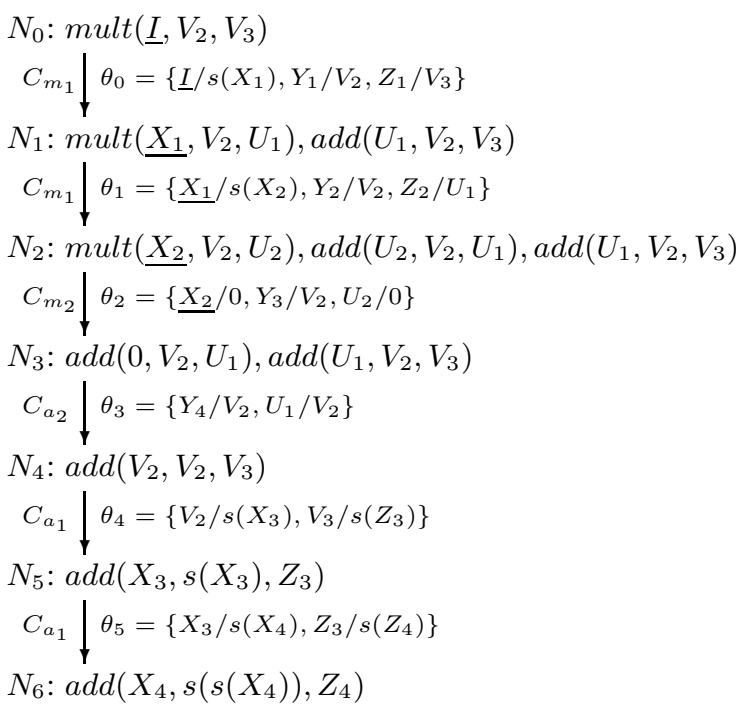

(a)

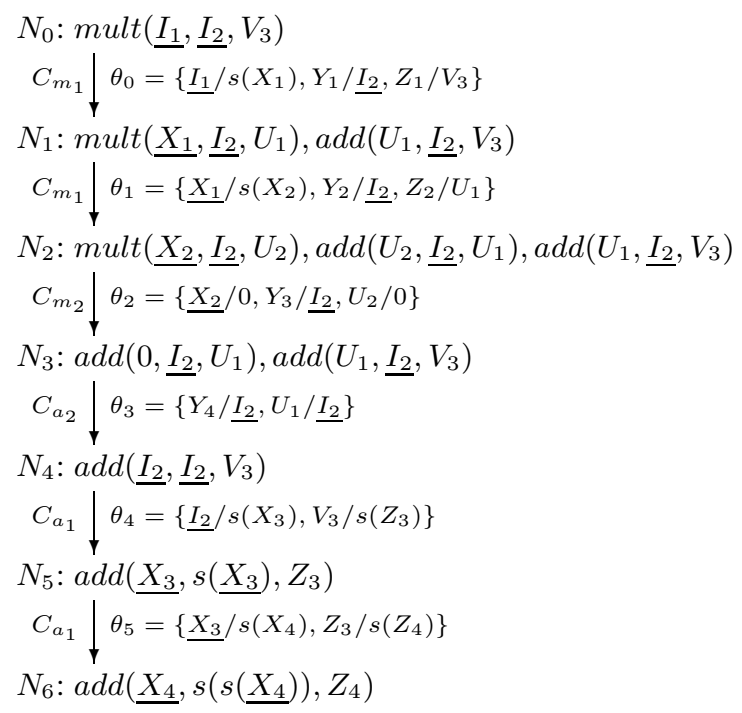

(b)

Figure 7: Two moded generalized SLDNF-trees of $P_{3}$ generated by Algorithm 1.

AProVE07 [14], NTI [27, 28], Polytool [24, 25] and TALP [26] are four well-known state-ofthe-art analyzers. NTI proves non-termination, while the others prove termination. The Termination Competition 2007 [37] reports their latest performance. We borrow three representative logic programs from the competition website to further demonstrate the effectiveness of our termination prediction.

Example 10 Consider the following logic program coming from the Termination Competition 2007 with Problem id LP/talp/apt - subset1 and difficulty rating 100\%. AProVE07, NTI, Polytool and TALP all failed to prove/disprove its termination by yielding an answer "don't know" in the competition [37].

$$
\begin{array}{rlr}
P_{4}: & \text { member } 1(X,[Y \mid X s]) \leftarrow \text { member } 1(X, X s) . & C_{m_{1}} \\
& \text { member } 1(X,[X \mid X s]) . & C_{m_{2}} \\
& \text { subset } 1([X \mid X s], Y s): \text {-member } 1(X, Y s), \operatorname{subset} 1(X s, Y s) . & C_{s_{1}} \\
& \text { subset } 1([], Y s) . & C_{s_{2}}
\end{array}
$$

Query Mode: subset $1(o, i)$.

The query mode $\operatorname{subset} 1(o, i)$ means that the second argument of any query must be a ground term, while the first one can be an arbitrary term. Then, to prove the termination property of $P_{4}$ with this query mode is to prove the termination for the moded query $Q_{0}=\operatorname{subset} 1(V, \mathcal{I})$. Applying Algorithm 1 generates a moded generalized SLDNF-tree as shown in Figure 8. The prefix from 
$N_{0}$ down to $N_{3}$ satisfies the conditions of LP-check and has the term-size decrease property, so clause $C_{m_{1}}$ is skipped when expanding $N_{3}$. When the derivation is extended to $N_{10}$, the conditions of LP-check are satisfied again, where $G_{10}$ is a loop goal of $G_{9}$ that is a loop goal of $G_{8}$. Since the derivation has the term-size decrease property, $N_{10}$ is expanded by $C_{m_{2}}$.

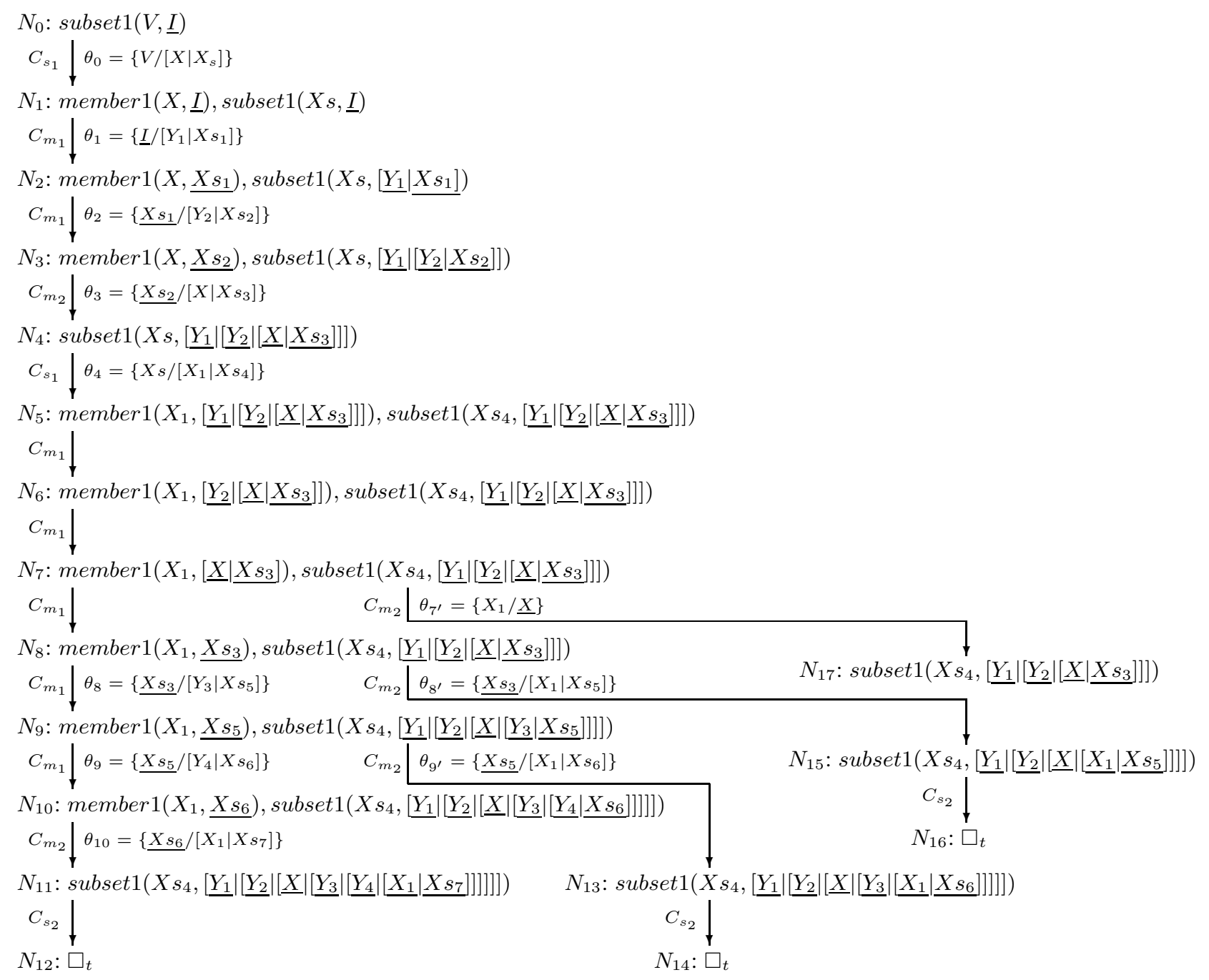

Figure 8: The moded generalized SLDNF-tree of $P_{4}$ generated by Algorithm 1.

At $N_{11}$ (resp. $N_{13}$ and $N_{15}$ ), the derivation satisfies the conditions of LP-check and has the term-size decrease property, where $G_{11}$ (resp. $N_{13}$ and $N_{15}$ ) is a loop goal of $G_{4}$ that is a loop goal of $G_{0}$. Therefore, $N_{11}$ (resp. $N_{13}$ and $N_{15}$ ) is expanded by $C_{s_{2}}$. When the derivation is extended to $N_{17}$, the conditions of LP-check are satisfied, where $G_{17}$ is a loop goal of $G_{4}$ that is a loop goal of $G_{0}$, but the term-size decrease condition is violated. Algorithm 1 stops immediately with an answer, predicted-non-terminating, for the query $Q_{0}$. It is easy to verify that this prediction is correct. 
Example 11 Consider another logic program in the Termination Competition 2007 with Problem id LP/SGST06 - incomplete and difficulty rating $75 \%$. Polytool succeeded to prove its termination, while AProVE07, NTI and TALP failed [37].

$$
\begin{array}{rlr}
P_{5}: & p(X) \leftarrow q(f(Y)), p(Y) . & \\
& p(g(X)) \leftarrow p(X) . & C_{p_{1}} \\
& q(g(Y)) . & C_{p_{2}} \\
& & C_{q_{1}}
\end{array}
$$

Query Mode: $p(i)$.

To prove the termination property of $P_{5}$ with this query mode is to prove the termination for the moded query $Q_{0}=p(\mathcal{I})$. Applying Algorithm 1 generates a moded generalized SLDNF-tree as shown in Figure 9. The prefix from $N_{0}$ down to $N_{4}$ satisfies the conditions of LP-check and has the term-size decrease property, so clause $C_{p_{2}}$ is skipped when expanding $N_{4}$. Algorithm 1 yields an answer predicted-terminating for the query $Q_{0}$. This prediction is correct.

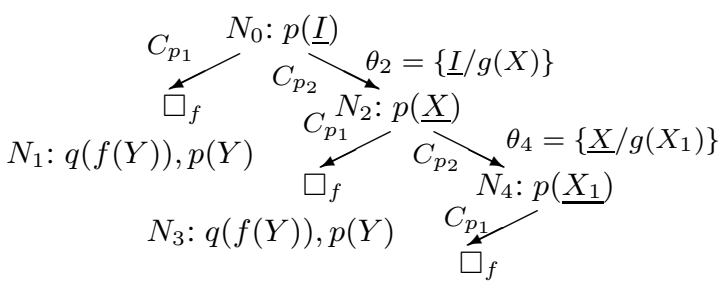

$$
\begin{aligned}
& N_{5}: q(f(Y)), p(Y)
\end{aligned}
$$

Figure 9: The moded generalized SLDNF-tree of $P_{5}$ generated by Algorithm 1.

Example 12 Consider a third logic program from the Termination Competition 2007 with Problem id LP/SGSTO6 - incomplete 2 and difficulty rating $75 \%$. In contrast to Example 11, for this program AProVE07 succeeded to prove its termination, while Polytool, NTI and TALP failed [37].

$$
\begin{aligned}
P_{6}: & f(X) \leftarrow g(s(s(s(X)))) . & & C_{f_{1}} \\
& f(s(X)) \leftarrow f(X) . & & C_{f_{2}} \\
& g(s(s(s(s(X)))) \leftarrow f(X) . & & C_{g_{1}}
\end{aligned}
$$

Query Mode: $f(i)$.

To prove the termination property of $P_{6}$ with this query mode is to prove the termination for the moded query $Q_{0}=f(\mathcal{I})$. Applying Algorithm 1 generates a moded generalized SLDNFtree as shown in Figure 10. $C_{f_{1}}$ and/or $C_{f_{2}}$ is skipped at $N_{4}, N_{5}, N_{6}, N_{9}, N_{10}, N_{11}, N_{13}, N_{18}$, $N_{19}, N_{20}, N_{22}, N_{23}, N_{25}$ and $N_{27}$, due to the occurrence of the following prefixes which satisfy both the conditions of LP-check and the term-size decrease condition: 
1. $\quad N_{0}: f(\underline{I}) \Rightarrow_{C_{f_{1}}} \ldots N_{2}: f(\underline{X}) \Rightarrow_{C_{f_{1}}} \ldots N_{4}: f\left(\underline{X_{1}}\right) \Rightarrow_{C_{f_{1}}}$

2. $\quad N_{0}: f(\underline{I}) \Rightarrow_{C_{f_{1}}} \ldots N_{2}: f(\underline{X}) \Rightarrow_{C_{f_{1}}} \ldots N_{5}: f\left(\underline{X_{2}}\right) \Rightarrow_{C_{f_{1}}}$

3. $\quad N_{0}: f(\underline{I}) \Rightarrow_{C_{f_{1}}} \ldots N_{2}: f(\underline{X}) \Rightarrow_{C_{f_{1}}} \ldots N_{6}: f\left(\underline{X_{3}}\right) \Rightarrow_{C_{f_{1}}}$

4. $\quad N_{0}: f(\underline{I}) \Rightarrow_{C_{f_{1}}} \ldots N_{4}: f\left(\underline{X_{1}}\right) \Rightarrow_{C_{f_{2}}} N_{5}: f\left(\underline{X_{2}}\right) \Rightarrow_{C_{f_{2}}} N_{6}: f\left(\underline{X_{3}}\right) \Rightarrow_{C_{f_{2}}}$

5. $\quad N_{0}: f(\underline{I}) \Rightarrow_{C_{f_{1}}} \ldots N_{7}: f\left(\underline{X_{1}}\right) \Rightarrow_{C_{f_{1}}} \ldots N_{9}: f\left(\underline{X_{2}}\right) \Rightarrow_{C_{f_{1}}}$

6. $\quad N_{0}: f(\underline{I}) \Rightarrow_{C_{f_{1}}} \ldots N_{7}: f\left(\underline{X_{1}}\right) \Rightarrow_{C_{f_{1}}} \ldots N_{10}: f\left(\underline{X_{3}}\right) \Rightarrow_{C_{f_{1}}}$

7. $\quad N_{0}: f(\underline{I}) \Rightarrow_{C_{f_{1}}} \ldots N_{2}: f(\underline{X}) \Rightarrow_{C_{f_{2}}} \ldots N_{9}: f\left(\underline{\left.X_{2}\right)} \Rightarrow_{C_{f_{2}}} N_{10}: f\left(\underline{X_{3}}\right) \Rightarrow_{C_{f_{2}}}\right.$

8. $\quad N_{0}: f(\underline{I}) \Rightarrow_{C_{f_{1}}} \ldots N_{11}: f\left(\underline{X_{2}}\right) \Rightarrow_{C_{f_{1}}} \ldots N_{13}: f\left(\underline{X_{3}}\right) \Rightarrow_{C_{f_{1}}}$

9. $\quad N_{0}: f(\underline{I}) \Rightarrow_{C_{f_{1}}} \ldots N_{2}: f(\underline{X}) \Rightarrow_{C_{f_{2}}} N_{7}: f\left(\underline{X_{1}}\right) \Rightarrow_{C_{f_{2}}} \ldots N_{13}: f\left(\underline{X_{3}}\right) \Rightarrow_{C_{f_{2}}}$

10. $N_{0}: f(\underline{I}) \Rightarrow_{C_{f_{1}}} \ldots N_{2}: f(\underline{X}) \Rightarrow_{C_{f_{2}}} N_{7}: f\left(\underline{X_{1}}\right) \Rightarrow_{C_{f_{2}}} N_{11}: f\left(\underline{X_{2}}\right) \Rightarrow_{C_{f_{2}}}$

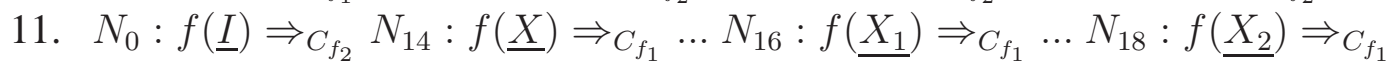

12. $N_{0}: f(\underline{I}) \Rightarrow_{C_{f_{2}}} N_{14}: f(\underline{X}) \Rightarrow_{C_{f_{1}}} \ldots N_{16}: f\left(\underline{X_{1}}\right) \Rightarrow_{C_{f_{1}}} \ldots N_{19}: f\left(\underline{X_{3}}\right) \Rightarrow_{C_{f_{1}}}$

13. $N_{0}: f(\underline{I}) \Rightarrow_{C_{f_{2}}} \ldots N_{18}: f\left(\underline{X_{2}}\right) \Rightarrow_{C_{f_{2}}} N_{19}: f\left(\underline{X_{3}}\right) \Rightarrow_{C_{f_{2}}}$

14. $N_{0}: f(\underline{I}) \Rightarrow_{C_{f_{2}}} N_{14}: f(\underline{X}) \Rightarrow_{C_{f_{1}}} \ldots N_{20}: f\left(\underline{X_{2}}\right) \Rightarrow_{C_{f_{1}}} \ldots N_{22}: f\left(\underline{X_{3}}\right) \Rightarrow_{C_{f_{1}}}$

15. $N_{0}: f(\underline{I}) \Rightarrow_{C_{f_{2}}} \ldots N_{16}: f\left(\underline{X_{1}}\right) \Rightarrow_{C_{f_{2}}} \ldots N_{22}: f\left(\underline{X_{3}}\right) \Rightarrow_{C_{f_{2}}}$

16. $N_{0}: f(\underline{I}) \Rightarrow_{C_{f_{2}}} \ldots N_{16}: f\left(\underline{X_{1}}\right) \Rightarrow_{C_{f_{2}}} N_{20}: f\left(\underline{X_{2}}\right) \Rightarrow_{C_{f_{2}}}$

17. $N_{0}: f(\underline{I}) \Rightarrow_{C_{f_{2}}} \ldots N_{23}: f\left(\underline{X_{1}}\right) \Rightarrow_{C_{f_{1}}} \ldots N_{25}: f\left(\underline{X_{2}}\right) \Rightarrow_{C_{f_{1}}} \ldots N_{27}: f\left(\underline{X_{3}}\right) \Rightarrow_{C_{f_{1}}}$

18. $\quad N_{0}: f(\underline{I}) \Rightarrow_{C_{f_{2}}} N_{14}: f(\underline{X}) \Rightarrow_{C_{f_{2}}} \ldots N_{27}: f\left(\underline{X_{3}}\right) \Rightarrow_{C_{f_{2}}}$

19. $N_{0}: f(\underline{I}) \Rightarrow_{C_{f_{2}}} N_{14}: f(\underline{X}) \Rightarrow_{C_{f_{2}}} \ldots N_{25}: f\left(\underline{X_{2}}\right) \Rightarrow_{C_{f_{2}}}$

20. $N_{0}: f(\underline{I}) \Rightarrow_{C_{f_{2}}} N_{14}: f(\underline{X}) \Rightarrow_{C_{f_{2}}} \ldots N_{23}: f\left(\underline{X_{1}}\right) \Rightarrow_{C_{f_{2}}}$

Since there is no derivation satisfying the conditions of LP-check while violating the term-size decrease condition, Algorithm 1 ends with an answer predicted-terminating for the query $Q_{0}$. This again is a correct prediction.

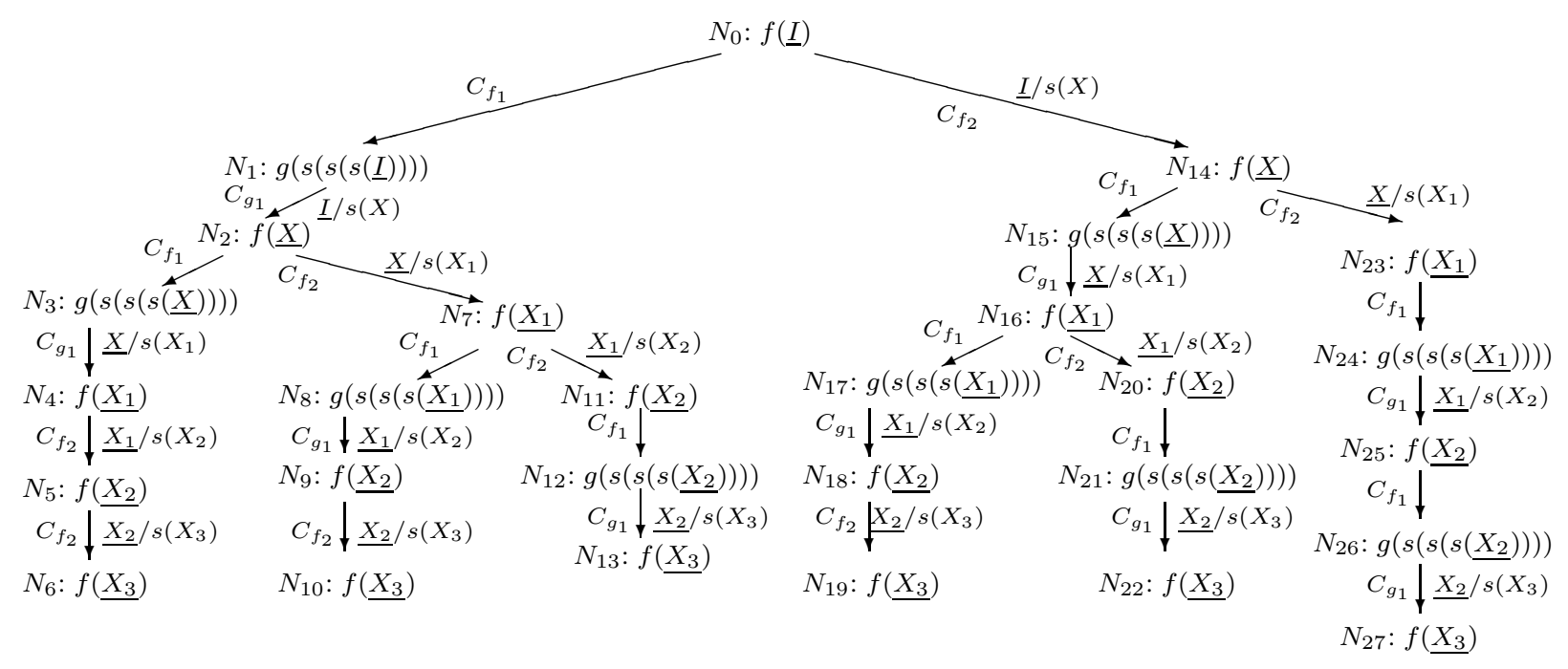

Figure 10: The moded generalized SLDNF-tree of $P_{6}$ generated by Algorithm 1. 
Choosing $r=3$ for Algorithm 1, we are able to obtain correct predictions for all benchmark programs of the Termination Competition 2007 (see Section 6). However, we should remark that due to the undecidability of the termination problem, there exist cases that choosing $r=3$ will lead to an inncorect prediction. Consider the following carefully crafted logic program:

$$
\begin{array}{lll}
P_{7}: & p(f(X), Y) \leftarrow p(X, s(Y)) . & C_{p_{1}} \\
& p(Z, \underbrace{s(s(\ldots s}_{100 \text { items }}(0) \ldots))) \leftarrow q . & C_{p_{2}} \\
& q \leftarrow q . & C_{q_{1}}
\end{array}
$$

$P_{7}$ does not terminate for a moded query $Q_{0}=p(\mathcal{I}, 0)$, as there is an infinite derivation

$$
N_{0}: p(\underline{I}, 0) \Rightarrow_{C_{p_{1}}} \ldots N_{101}: q \Rightarrow_{C_{q_{1}}} N_{102}: q \Rightarrow_{C_{q_{1}}} \ldots
$$

(see Figure 11) which satisfies conditions (i) and (ii) of Theorem 3, where for any $j \geq 101, G_{j+1}$ is a loop goal of $G_{j}$. Note that for any repetition number $r$ with $3 \leq r \leq 100$, the prefix ending at $N_{r-1}$ satisfies both the conditions of LP-check and the term-size decrease property, where for any $j$ with $0 \leq j<r-1, G_{j+1}$ is a loop goal of $G_{j}$. However, for any $r>100$, a prefix ending at $N_{100+r}$ will be encountered, which satisfies the conditions of LP-check but violates the term-size decrease condition, where for any $j$ with $101 \leq j<100+r, G_{j+1}$ is a loop goal of $G_{j}$. Therefore, Algorithm 1 will return predicted-terminating for $Q_{0}$ unless $r$ is set above 100.

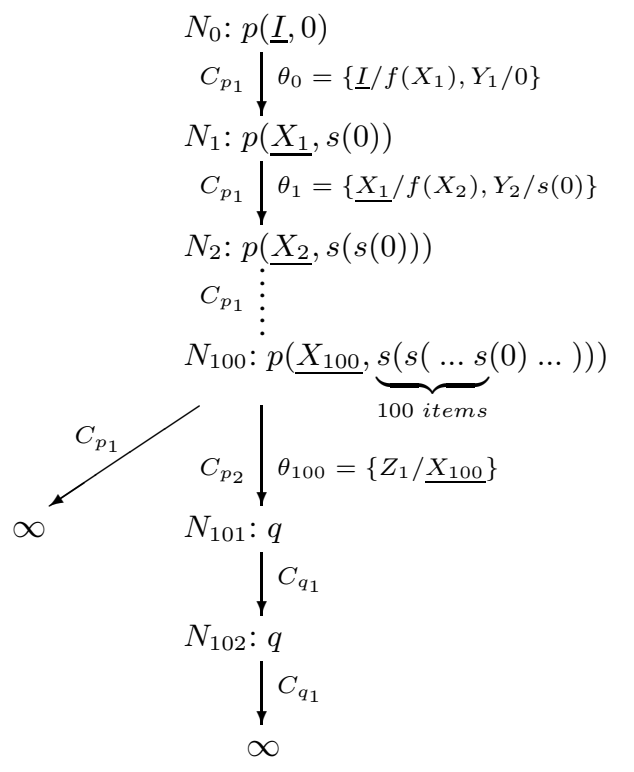

Figure 11: The moded generalized SLDNF-tree of $P_{7}$ with a moded query $p(\mathcal{I}, 0)$.

The following result shows that choosing a sufficiently large repetition number guarantees the correct prediction for non-terminating programs. 
Theorem 9 Let $P$ be a logic program and $Q$ be a query such that $P$ is non-terminating for $Q$. There always exists a number $R$ such that Algorithm 1 with any repetition number $r \geq R$ produces the answer predicted-non-terminating.

Proof: Let us assume the contrary. That is, we assume that for any number $N$, there exists a larger number $r$ such that Algorithm 1 for $P$ with query $Q$ and repetition number $r$ produces the answer predicted-terminating. When $N \rightarrow \infty$, this means that the prefix of form (3) of each infinite branch $D$ in the moded generalized SLDNF-tree $M T_{Q}$ satisfies the term-size decrease property. According to Theorem $6, D$ has an infinite chain of substitutions of form (1) for some input variable $I$ at $Q$. This means that $D$ does not satisfy condition (ii) of Theorem 3. However, since $P$ is non-terminating for $Q$, by Corollary $1 M T_{Q}$ has at least one infinite branch of form (2) satisfying conditions (i) and (ii) of Theorem 3 . We then have a contradiction and thus conclude the proof.

\section{Experimental Results}

We have evaluated our termination prediction technique on a benchmark of 301 Prolog programs. In this section, we first describe the benchmark and our experimental results using a straightforward implementation of Algorithm 1. Then, we define a pruning technique to reduce the size of moded generalized SLDNF-trees generated for our prediction. Finally, we make a comparison between the state-of-the-art termination and non-termination analyzers and our termination prediction tool.

Our benchmark consists of 301 programs with moded queries from the termination competition of 2007 [37]. Only 23 programs of the competition are omitted because they contain nonlogical operations such as arithmetics (for most of these programs neither termination nor nontermination could be shown by any of the tools in the competition). The benchmark contains 244 terminating programs and 57 non-terminating ones. The most accurate termination analyzer of the competition, AProVE [14], proves termination of 238 benchmark programs. The non-termination analyzer NTI $[27,28]$ proves non-termination of 42 programs. Because the prediction does not produce a termination or non-termination proof, our goal is to outperform the analyzers of the competition by providing more correct predictions.

We implemented our tool, TPoLP: Termination Prediction of Logic Programs, in SWI-prolog [36]. TPoLP is freely available from [38]. The moded generalized SLDNF-tree is generated following Algorithm 1. It is initialized with the moded query and extended until all branches are cut or a timeout occurs. To improve the efficiency of the analysis, a number of optimalizations were implemented, such as constant time access to the nodes and the arcs of the derivations. The experiments have been performed using SWI-Prolog 5.6.40, on an Intel Core2 Duo 2,33GHz, 2 Gb RAM.

Table 1 gives an overview of the predictions with repetition numbers $r=2, r=3$, and $r=4$. As we mentioned earlier, $r=2$ does not suffice because some of the predictions are wrong and we 
want high reliable predictions. When $r$ is set above two, all predictions made for the benchmark are correct. This shows that in practice, there is no need to increase the repetition number any further.

\begin{tabular}{|c|c|c|c|}
\hline Repetition number $r:$ & 2 & 3 & 4 \\
\hline Correct predictions: & 291 & 271 & 234 \\
Wrong predictions: & 7 & 0 & 0 \\
Out of time/memory & 3 & 30 & 67 \\
Average time (Sec): & 1.7 & 24.9 & 59.3 \\
\hline
\end{tabular}

Table 1: Predictions: full table available at [38]

When we increase the repetition number, the cost of prediction increases as well. Table 1 shows that for $r=3$, about $10 \%$ of the programs break the time limit of four minutes, and for $r=4$, about $20 \%$ break the limit.

The component of the algorithm taking most of the time differs from program to program. When a lot of branches are cut by LP-check, constructing the LP cuts is usually the bottleneck. For programs with a low amount of LP cuts, most of the time is spent on constructing the SLDNFderivations. Some of the derivations count more than a million nodes. To overcome such performance issues, we implemented the following pruning technique on loop goals.

Definition 9 (Pruning variants) Let $G_{2}$ be a loop goal of $G_{1}$ for which the selected subgoals are variants. Then, all clauses that have already been applied at $G_{2}$ are skipped at $G_{1}$ during backtracking.

The idea of this pruning is simple. For loop goals with variant selected subgoals, applying the non-looping clauses to them will generate the same derivations below them with the same termination properties. Therefore, the derivations already generated below $G_{2}$ need not be regenerated at $G_{1}$ during backtracking.

For the sake of efficiency, in our implementation we determine variants by checking that they have the same symbol string.

Consider Example 12 again. When the above pruning mechanism is applied, Algorithm 1 will simplify the moded generalized SLDNF-tree of Figure 10 into Figure 12. The pruning takes place at $N_{2}$ and $N_{0}$, where $G_{4}$ is a loop goal of $G_{2}$ that is a loop goal of $G_{0}$ and their selected subgoals are variants.

A stronger version of the above pruning mechanism can be obtained by removing the condition in Definition 9: for which the selected subgoals are variants. That is, we do not require the selected subgoals of loop goals to be variants. We call this version Pruning loop goals.

Table 2 gives an overview of our predictions with $r=3$ as the repetition number in the cases of no pruning, pruning variants, and pruning loop goals. The table shows that pruning is a good 


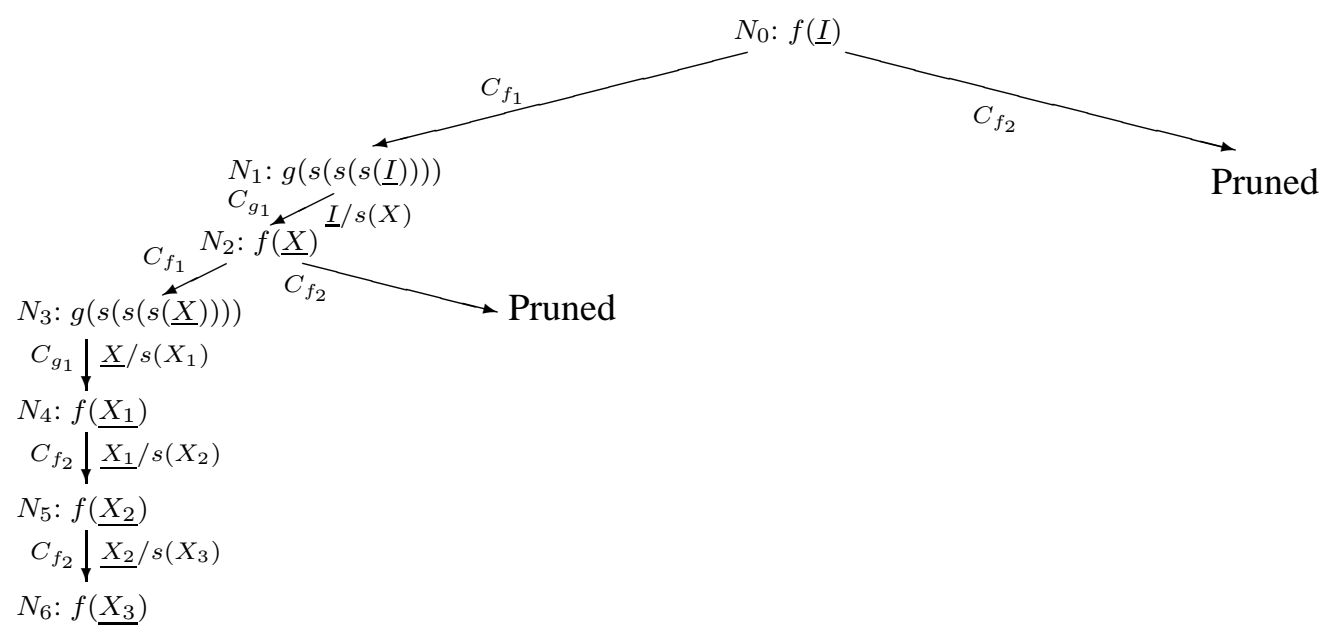

Figure 12: Figure 10 is simplified with pruning

tradeoff between the accuracy and the efficiency of the prediction. When applying the variants pruning mechanism the size of the derivations drops considerably, while all predictions for the benchmark are still correct. Due to the pruning, more than $98 \%$ of the predictions finish within the time limit. Applying the loop goals pruning mechanism leads to a greater reduction in the size of derivations. However, in this case we sacrifice accuracy: three non-terminating programs are predicted to be terminating.

\begin{tabular}{|r|c|c|c|}
\hline & No pruning & Pruning variants & Pruning loop goals \\
\hline Correct predictions: & 271 & 296 & 297 \\
Wrong predictions: & 0 & 0 & 3 \\
Out of time/memory & 30 & 5 & 1 \\
Average time (Sec) & 24.9 & 4.4 & 0.05 \\
\hline
\end{tabular}

Table 2: Pruning: full tables available at [38].

Table 3 gives a comparison between our predictions (with $r=3$ and the variants pruning mechanism) and the state-of-the-art termination and non-termination analyzers. Note that our tool, TPoLP, is the only tool which analyzes both for termination and non-termination of logic programs. The results are very encouraging. We correctly predict the termination property of all benchmark programs except for five programs which broke the time limit. It is also worth noticing that for all programs of the benchmark, either an existing analyzer finds a termination or non-termination proof, or a correct prediction is made by our tool. This shows that our prediction tool can be a very useful addition to any termination or non-termination analyzer. 


\begin{tabular}{|r|c|c|c|c|c|}
\hline & TPoLP & AProVE [14] & NTI [27, 28] & Polytool & TALP [26] \\
\hline Answer Terminating (244): & 239 & 238 & 0 & 206 & 164 \\
Answer Non-terminating (57): & 57 & 0 & 42 & 0 & 0 \\
\hline
\end{tabular}

Table 3: Comparison between TPoLP and the existing analyzers.

\section{Related Work}

Most existing approaches to the termination problem are norm- or level mapping-based in the sense that they perform termination analysis by building from the source code of a logic program some well-founded termination conditions/constraints in terms of norms (i.e. term sizes of atoms of clauses), level mappings, interargument size relations and/or instantiation dependencies, which when solved, yield a termination proof (see, e.g., [10] for a survey and more recent papers $[2,5,6,11,13,16,19,23])$. Another main stream is transformational approaches, which transform a logic program into a term rewriting system (TRS) and then analyze the termination property of the resulting TRS instead [1, 3, 14, 20, 26, 29, 31, 41]). All of these approaches are used for a termination proof; i.e., they compute sufficient termination conditions which once satisfied, lead to a positive conclusion terminating. Recently, Payet and Mesnard [27, 28] propose an approach to computing sufficient non-termination conditions which when satisfied, lead to a negative conclusion non-terminating. A majority of these termination/non-termination proof approaches apply only to positive logic programs.

Our approach presented in this paper differs significantly from existing termination analysis approaches. First, we do not make a termination proof, nor do we make a non-termination proof. Instead, we make a termination prediction (see Figure 1) - an approximation approach to attacking the undecidable termination problem. Second, we do not rely on static norms or level mappings, nor do we transform a logic program to a term rewriting system. Instead, we focus on detecting infinite SLDNF-derivations with the understanding that a logic program is terminating for a query if and only if there is no infinite SLDNF-derivation with the query. We have established a necessary and sufficient characterization of infinite SLDNF-derivations with arbitrary (concrete or moded) queries, introduced a new loop checking mechanism, and developed an algorithm that predicts termination of general logic programs with arbitrary queries by identifying potential infinite SLDNF-derivations. Since the algorithm implements the necessary and sufficient conditions (the characterization) of an infinite SLDNF-derivation, its prediction is very effective. Our experimental results show that except for five programs which break the time limit, our prediction is $100 \%$ correct for all benchmark programs of the Termination Competition 2007, of which eighteen programs cannot be proved by the existing state-of-the-art analyzers like AProVE07 [14], NTI [27, 28], Polytool [24, 25] and TALP [26].

Our termination prediction approach uses a loop checking mechanism (a loop check) to implement a characterization of infinite SLDNF-derivations. Well-known loop checks include VA-check 
[4, 39], OS-check [7, 21, 30], and VAF-checks [32, 33]. All apply to positive logic programs. In particular, VA-check applies to function-free logic programs, where an infinite derivation is characterized by a sequence of selected variant subgoals. OS-check identifies an infinite derivation with a sequence of selected subgoals with the same predicate symbol whose sizes do not decrease. VAF-checks take a sequence of selected expanded variant subgoals as major characteristics of an infinite derivation. Expanded variant subgoals are variant subgoals except that some terms may grow bigger. In this paper, a new loop check mechanism, LP-check, is introduced in which an infinite derivation is identified with a sequence of loop goals. Most importantly, enhancing LP-check with the term-size decrease property leads to the first loop check for moded queries.

\section{Conclusion and Future Work}

We have presented an approximation framework for attacking the undecidable termination problem of logic programs, as an alternative to current termination/non-termination proof approaches. We introduced an idea of termination prediction, established a necessary and sufficient characterization of infinite SLDNF-derivations with arbitrary (concrete or moded) queries, built a new loop checking mechanism, and developed an algorithm that predicts termination of general logic programs with arbitrary queries. We have implemented a termination prediction tool, TPoLP, and obtained quite satisfactory experimental results. Except for five programs which break the experiment time limit, our prediction is $100 \%$ correct for all benchmark programs of the Termination Competition 2007.

Our prediction approach can be used standalone, e.g., it may be incorporated into Prolog as a termination debugging tool; or it is used along with some termination/non-termination proof tools (see the framework in Figure 1).

Limitations of the current prediction approach include that it cannot handle floundering queries and programs with non-logical operators.

Our future work includes further improvement of the prediction efficiency of TPoLP. As shown in Table 2, there are five benchmark programs breaking our experiment time limit. We are also considering extensions of the proposed termination prediction to typed queries [6] and to logic programs with tabling $[8,35,40]$.

\section{References}

[1] G. Aguzzi and U. Modigliani, Proving termination of logic programs by transforming them into equivalent term rewriting systems, Proc. of the 13th FST and TCS, LNCS 761, 1993, pp. $114-124$. 
[2] K. R. Apt and D. Pedreschi, Reasoning about termination of pure Prolog programs, Information and Computation 106(1):109-157 (1993).

[3] T. Arts and H. Zantema, Termination of logic programs using semantic unification, Proc. of the 5th International Symposium on Logic-based Program Synthesis and Transformation, 1995, pp. 219-233.

[4] R. N. Bol, K. R. Apt and J. W. Klop, An analysis of loop checking mechanisms for logic programs, Theoretical Computer Science 86(1):35-79 (1991).

[5] A. Bossi, N. Cocco, S. Rossi and S. Etalle, On modular termination proofs of general logic programs, Theory and Practice of Logic Programming 2(3):263-291 (2002).

[6] M. Bruynooghe, M. Codish, J. Gallagher, S. Genaim, and W. Vanhoof, Termination analysis of logic programs through combination of type-based norms, ACM Transactions on Programming Languages and Systems, to appear.

[7] M. Bruynooghe, D. De Schreye and B. Martens, A general criterion for avoiding infinite unfolding during partial deduction, New Generation Computing 11(1):47-79 (1992).

[8] W. D. Chen and D. S. Warren, Tabled evaluation with delaying for general logic programs, $J$. ACM 43(1):20-74 (1996).

[9] K. L. Clark, Negation as Failure, in: (H. Gallaire and J. Minker, eds.) Logic and Databases, Plenum, New York, 1978, pp. 293-322.

[10] D. De Schreye and S. Decorte, Termination of logic programs: the never-ending story, Journal of Logic Programming 19/20:199-260 (1993).

[11] S. Decorte, D. De Schreye and H. Vandecasteele, Constraint-based termination analysis of logic programs, ACM Transactions on Programming Languages and Systems 21(6):11371195 (1999).

[12] N. Dershowttz, N. Lindenstrauss, Y. Sagiv and A. Serebrenik, A general framework for automatic termination analysis of logic programs, Applicable Algebra in Engineering, Communication and Computing 12(1/2):117-156 (2001).

[13] S. Genaim and M. Codish, Inferring termination conditions for logic programs using backwards analysis, Theory and Practice of Logic Programming 5(1/2):75-91 (2005).

[14] J. Giesl, P. Schneider-Kamp and R. Thiemann, AProVE 1.2: Automatic termination proofs in the DP framework, Proc. of the 3rd IJCAR, LNAI 4130, 2006, pp. 281-286.

[15] ISLAB, SICStus Prolog User's Manual, Intelligent Systems Laboratory, Swedish Institute of Computer Science, 2008. Available at http://www.sics.se/sicstus/docs/latest4/pdf/sicstus.pdf. 
[16] N. Lindenstrauss and Y. Sagiv, Automatic termination analysis of logic programs, in: L. Naish (ed.) Proc. of the Fourteenth International Conference on Logic Programming, Leuven, Belgium. MIT Press, 1997, pp. 63-77.

[17] N. Lindenstrauss, Y. Sagiv and A. Serebrenik, TermiLog: a system for checking termination of queries to logic programs, Proc. of the 9th CAV, 1997, pp. 444-447.

[18] J. W. Lloyd, Foundations of Logic Programming, 2nd ed., Springer-Verlag, Berlin, 1987.

[19] E. Marchiori, Practical methods for proving termination of general logic programs, Journal of Artificial Intelligence Research 4:179-208 (1996).

[20] M. Marchiori, Proving existential termination of normal logic programs, Proc. of the 5th AMAST, LNCS 1101, 1996, pp. 375-390.

[21] B. Martens and D. De Schreye, Automatic finite unfolding using well-founded measures, Journal of Logic Programming 28(2):89-146 (1996).

[22] F. Mesnard and R. Bagnara, cTI: a constraint-based termination inference tool for ISOProlog, Theory and Practice of Logic Programming 5(1/2):243-257 (2005).

[23] F. Mesnard and U. Neumerkel, Applying static analysis techniques for inferring termination conditions of logic programs, The 8th International Symposium on Static Analysis, Paris, France, 2001, pp. 93-110.

[24] M. T. Nguyen and D. De Schreye, Polynomial interpretations as a basis for termination analysis of logic programs, Proc. of the 21st International Conference on Logic Programming, LNCS 3668, 2005, pp. 311-326.

[25] M. T. Nguyen, D. De Schreye, J. Giesl and P. Schneider-Kamp, Polytool: proving termination automatically based on polynomial interpretations, Technical Report, Department of Computer Science, K.U.Leuven, Belgium, 2006.

[26] E. Ohlebusch, C. Claves and C. Marche, TALP: a tool for the termination analysis of logic programs, Proc. of 11th RTA, LNCS 1833, 2000, pp. 270-273.

[27] E. Payet and F. Mesnard, Non-termination inference of logic programs, ACM Transactions on Programming Languages and Systems 28(2):256-289 (2006).

[28] E. Payet, Detecting non-termination of term rewriting systems using an unfolding operator, Proc. of the 16th International Symposium on Logic-Based Program Synthesis and Transformation, LNCS 4407, 2006, pp. 194-209.

[29] M. K. Rao, D. Kapur and R. Shyamasundar, Transformational methodology for proving termination of logic programs, Journal of Logic Programming 34(1):1-42 (1998). 
[30] D. Sahlin, Mixtus: an automatic partial evaluator for full Prolog, New Generation Computing 12(1):7-51 (1993).

[31] P. Schneider-Kamp, J. Giesl, A. Serebrenik and R. Thiemann, Automated termination analysis for logic programs by term rewriting, International Symposium on Logic-based Program Synthesis and Transformation, Venice, Italy, 2006, pp. 177-193.

[32] Y. D. Shen, An extended variant of atoms loop check for positive logic programs, New Generation Computing 15(2):187-204 (1997).

[33] Y. D. Shen, L. Y. Yuan and J. H. You, Loop checks for logic programs with functions, Theoretical Computer Science 266(1-2):441-461 (2001).

[34] Y. D. Shen, J. H. You, L. Y. Yuan, S. P. Shen and Q. Yang, A dynamic approach to characterizing termination of general logic programs, ACM Transactions on Computational Logic 4(4):417-430 (2003).

[35] Y. D. Shen, J. H. You and L. Y. Yuan, Enhancing global SLS-resolution with loop cutting and tabling mechanisms, Theoretical Computer Science 328(3):271-287(2004).

[36] SWI-Prolog, available at http://www.swi-prolog.org/.

[37] Termination competition 2007, available at http://www.lri.fr/ $\sim$ marche/terminationcompetition/2007.

[38] TPoLP: Termination Prediction of Logic Programs, available at http://www.cs.kuleuven.be/ dean.

[39] A. Van Gelder, Efficient loop detection in Prolog, Journal of Logic Programming 4:23-31 (1987).

[40] S. Verbaeten, D. D. Schreye and K. Sagonas, Termination proofs for logic programs with tabling, ACM Transactions on Computational Logic 2(1):57-92 (2001).

[41] F. van Raamsdonk, Translating logic programs into conditional rewriting systems, Proc. of the 14th ICLP, 1997, pp. 168-182. 